\title{
Spitzer deep and wide legacy mid- and far-infrared number counts and lower limits of cosmic infrared background ${ }^{\star}$
}

\author{
M. Béthermin ${ }^{1}$, H. Dole ${ }^{1}$, A. Beelen ${ }^{1}$, and H. Aussel ${ }^{2}$ \\ ${ }^{1}$ Institut d'Astrophysique Spatiale (IAS), Bt. 121, 91405 Orsay, France; Université Paris-Sud 11 and CNRS (UMR8617) \\ e-mail: mattieu.bethermin@ias.u-psud.fr \\ ${ }^{2}$ Laboratoire AIM, CEA/DSM-CNRS-Université Paris Diderot, IRFU/Service d'Astrophysique, Bt. 709, CEA-Saclay, \\ 91191 Gif-sur-Yvette Cedex, France
}

Received 11 September 2009 / Accepted 5 January 2010

\section{ABSTRACT}

\begin{abstract}
Aims. We aim to place stronger lower limits on the cosmic infrared background (CIB) brightness at $24 \mu \mathrm{m}, 70 \mu \mathrm{m}$ and $160 \mu \mathrm{m}$ and measure the extragalactic number counts at these wavelengths in a homogeneous way from various surveys.

Methods. Using Spitzer legacy data over $53.6 \mathrm{deg}^{2}$ of various depths, we build catalogs with the same extraction method at each wavelength. Completeness and photometric accuracy are estimated with Monte-Carlo simulations. Number count uncertainties are estimated with a counts-in-cells moment method to take galaxy clustering into account. Furthermore, we use a stacking analysis to estimate number counts of sources not detected at $70 \mu \mathrm{m}$ and $160 \mu \mathrm{m}$. This method is validated by simulations. The integration of the number counts gives new CIB lower limits.

Results. Number counts reach $35 \mu \mathrm{Jy}, 3.5 \mathrm{mJy}$ and $40 \mathrm{mJy}$ at $24 \mu \mathrm{m}, 70 \mu \mathrm{m}$, and $160 \mu \mathrm{m}$, respectively. We reach deeper flux densities of $0.38 \mathrm{mJy}$ at 70 , and 3.1 at $160 \mu \mathrm{m}$ with a stacking analysis. We confirm the number count turnover at $24 \mu \mathrm{m}$ and $70 \mu \mathrm{m}$, and observe it for the first time at $160 \mu \mathrm{m}$ at about $20 \mathrm{mJy}$, together with a power-law behavior below $10 \mathrm{mJy}$. These mid- and far-infrared counts: 1) are homogeneously built by combining fields of different depths and sizes, providing a legacy over about three orders of magnitude in flux density; 2) are the deepest to date at $70 \mu \mathrm{m}$ and $160 \mu \mathrm{m}$; 3) agree with previously published results in the common measured flux density range; 4) globally agree with the Lagache et al. (2004) model, except at $160 \mu \mathrm{m}$, where the model slightly overestimates the counts around 20 and $200 \mathrm{mJy}$.

Conclusions. These counts are integrated to estimate new CIB firm lower limits of $2.29_{-0.09}^{+0.09} \mathrm{nW} \mathrm{m}^{-2} \mathrm{sr}^{-1}, 5.4_{-0.4}^{+0.4} \mathrm{nW} \mathrm{m}^{-2} \mathrm{sr}^{-1}$, and $8.9_{-1.1}^{+1.1} \mathrm{nW} \mathrm{m}^{-2} \mathrm{sr}^{-1}$ at $24 \mu \mathrm{m}, 70 \mu \mathrm{m}$, and $160 \mu \mathrm{m}$, respectively, and extrapolated to give new estimates of the CIB due to galaxies of $2.86_{-0.16}^{+0.19} \mathrm{nW} \mathrm{m}^{-2} \mathrm{sr}^{-1}, 6.6_{-0.6}^{+0.7} \mathrm{nW} \mathrm{m}^{-2} \mathrm{sr}^{-1}$, and $14.6_{-2.9}^{+7.1} \mathrm{nW} \mathrm{m}^{-2} \mathrm{sr}^{-1}$, respectively. Products (point spread function, counts, CIB contributions, software) are publicly available for download at http://wWw.ias.u-psud.fr/irgalaxies/
\end{abstract}

Key words. cosmology: observations - diffuse radiation - galaxies: statistics - galaxies: evolution - galaxies: photometry infrared: galaxies

\section{Introduction}

The extragalactic background light (EBL) is the relic emission of all processes of structure formation in the Universe. About half of this emission, called the Cosmic Infrared Background (CIB) is emitted in the $8-1000 \mu \mathrm{m}$ range, and peaks around $150 \mu \mathrm{m}$. It is essentially due to the star formation (Puget et al. 1996; Fixsen et al. 1998; Hauser et al. 1998; Lagache et al. 1999; Gispert et al. 2000; Hauser \& Dwek 2001; Kashlinsky 2005; Lagache et al. 2005).

The CIB spectral energy distribution (SED) is an important constraint for the infrared galaxies evolution models (e.g. Lagache et al. 2004; Franceschini et al. 2010; Le Borgne et al. 2009; Pearson \& Khan 2009; Rowan-Robinson 2009; Valiante et al. 2009). It gives the budget of infrared emission since the first star. The distribution of the flux of sources responsible for this background is also a critical constraint. We propose to measure the level of the CIB and the flux distribution of the sources at 3 wavelengths $(24 \mu \mathrm{m}, 70 \mu \mathrm{m}$ and $160 \mu \mathrm{m})$.

* Counts and CIB contributions are only available in electronic form at the CDS via anonymous ftp to

cdsarc.u-strasbg.fr $(130.79 .128 .5)$ or via

http://cdsweb.u-strasbg.fr/cgi-bin/qcat?J/A+A/512/A78
In the 1980's, the infrared astronomical satellite (IRAS) and COBE/DIRBE performed the first mid-infrared (MIR) and far-infrared (FIR) full-sky surveys. Nevertheless, the detected sources were responsible for a very small part of the CIB. Between 1995 and 1998, the ISO (infrared space observatory) performed deeper observations of infrared galaxies. Elbaz et al. (2002) resolved into the source more than half of the CIB at $15 \mu \mathrm{m}$. At larger wavelengths, the sensitivity and angular resolution was not sufficient to resolve the CIB (Dole et al. 2001).

The Spitzer space telescope (Werner et al. 2004), launched in 2003, has performed deep infrared observations on wide fields. The multiband imaging photometers for Spitzer (MIPS) (Rieke et al. 2004) mapped the sky at $24 \mu \mathrm{m}, 70 \mu \mathrm{m}$ and $160 \mu \mathrm{m}$. About $60 \%$ of the CIB was resolved at $24 \mu \mathrm{m}$ (Papovich et al. 2004) and at $70 \mu \mathrm{m}$ (Frayer et al. 2006). Because of confusion (Dole et al. 2003), only about $7 \%$ were resolved at $160 \mu \mathrm{m}$ (Dole et al. 2004). Dole et al. (2006) managed to resolve most of the $70 \mu \mathrm{m}$ and $160 \mu \mathrm{m}$ by stacking $24 \mu \mathrm{m}$ sources.

The cold mission of Spitzer is over, and lots of data are now public. We present extragalactic number counts built homogeneously by combining deep and wide fields. The large sky surface used significantly reduces uncertainties on number counts. In order to obtain very deep FIR number counts, we used a 
A\&A 512, A78 (2010)

Table 1. Size, $80 \%$ completeness flux density, and calibration scaling factor (see Sect. 2.1) of the used fields.

\begin{tabular}{|c|c|c|c|c|c|c|c|c|c|}
\hline \multirow[t]{3}{*}{ Field name } & \multicolumn{3}{|c|}{ Surface area } & \multicolumn{3}{|c|}{$80 \%$ completeness flux } & \multicolumn{3}{|c|}{ Scaling factor } \\
\hline & $24 \mu \mathrm{m}$ & $70 \mu \mathrm{m}$ & $160 \mu \mathrm{m}$ & $24 \mu \mathrm{m}$ & $70 \mu \mathrm{m}$ & $160 \mu \mathrm{m}$ & $24 \mu \mathrm{m}$ & $70 \mu \mathrm{m}$ & $160 \mu \mathrm{m}$ \\
\hline & & $\operatorname{deg}^{2}$ & & $\mu \mathrm{Jy}$ & \multicolumn{2}{|c|}{$\mathrm{mJy}$} & & & \\
\hline FIDEL eCDFS & 0.23 & 0.19 & - & 60. & 4.6 & - & 1.0157 & 1 & - \\
\hline FIDEL EGS & 0.41 & - & 0.38 & 76. & - & 45. & 1.0157 & - & 0.93 \\
\hline COSMOS & 2.73 & 2.41 & 2.58 & 96. & 7.9 & 46. & 1 & 0.92 & 0.96 \\
\hline SWIRE LH & 10.04 & 11.88 & 11.10 & 282. & 25.4 & 92. & 1.0509 & 1.10 & 0.93 \\
\hline SWIRE EN1 & 9.98 & 9.98 & 9.30 & 261. & 24.7 & 94. & 1.0509 & 1.10 & 0.93 \\
\hline SWIRE EN2 & 5.36 & 5.34 & 4.98 & 267. & 26.0 & 90. & 1.0509 & 1.10 & 0.98 \\
\hline SWIRE ES1 & 7.45 & 7.43 & 6.71 & 411. & 36.4 & 130. & 1.0509 & 1.10 & 0.98 \\
\hline SWIRE CDFS & 8.42 & 8.28 & 7.87 & 281. & 24.7 & 88. & 1.0509 & 1.10 & 0.98 \\
\hline SWIRE XMM & 8.93 & - & - & 351. & - & - & 1.0509 & - & - \\
\hline Total & 53.55 & 45.51 & 42.91 & & & & & & \\
\hline
\end{tabular}

Notes. Some fields are not used at all wavelengths.

stacking analysis and estimate the level of the CIB in the three MIPS bands with them.

\section{Data, source extraction and photometry}

\subsection{Data}

We took the public Spitzer mosaics ${ }^{1}$ from different observation programs: the GOODS/FIDEL (PI: M. Dickinson), COSMOS (PI: D. Sanders) and SWIRE (PI: C. Lonsdale). We used only the central part of each field, which was defined by a cut of $50 \%$ of the median coverage for SWIRE fields and $80 \%$ for the other. The total area covers $53.6 \mathrm{deg}^{2}, 45.5 \mathrm{deg}^{2}, 42.9 \mathrm{deg}^{2}$ at $24 \mu \mathrm{m}$, $70 \mu \mathrm{m}$ and $160 \mu \mathrm{m}$ respectively. The surface of the deep fields (FIDEL, COSMOS) is about $3.5 \mathrm{deg}^{2}$. Some fields were not used at all wavelengths for different reasons: There is no public release of FIDEL CDFS data at $160 \mu \mathrm{m}$; the pixels of the EGS $70 \mu \mathrm{m}$ are not square; XMM is not observed at 70 and $160 \mu \mathrm{m}$. Table 1 summarises the field names, sizes and completenesses.

In 2006, new calibration factors were adopted for MIPS (Engelbracht et al. 2007; Gordon et al. 2007; Stansberry et al. 2007). The conversion factor from instrumental unit to $\mathrm{MJy} / \mathrm{sr}$ is 0.0454 (resp. 702 and 41.7) at $24 \mu \mathrm{m}$ (resp. $70 \mu \mathrm{m}$ and $160 \mu \mathrm{m}$ ). The COSMOS GO3 and SWIRE (released 22 Dec. 2006) mosaics were generated with the new calibration. The FIDEL mosaics were obtained with other factors at $24 \mu \mathrm{m}$ and $160 \mu \mathrm{m}$ (resp. 0.0447 and 44.7). The $70 \mu \mathrm{m}$ and $160 \mu \mathrm{m}$ COSMOS mosaics were color corrected (see Sect. 2.3). Consequently we applied a scaling factor (see Table 1) before the source extraction to each mosaic to work on a homogeneous sample of maps (new calibration and no color correction).

\subsection{Source extraction and photometry}

The goal is to build homogeneous number counts with wellcontrolled systematics and high statistics. However, the fields present various sizes and depths. We thus employed a single extraction method at a given wavelength, allowing the heterogeneous datasets to combine in a coherent way.

\subsubsection{Mid-IR/far-IR differences}

The MIR $(24 \mu \mathrm{m})$ and FIR $(70 \mu \mathrm{m}$ and $160 \mu \mathrm{m})$ maps have different properties: in the MIR, we observe lots of faint blended

\footnotetext{
1 from the Spitzer Science Center website: http://data. spitzer. caltech.edu/popular/
}

sources; in the FIR, due to confusion (Dole et al. 2004), all these faint blended sources are only seen as background fluctuations. Consequently, we used different extraction and photometry methods for each wavelength. In the MIR, the priority is the deblending: accordingly we took the SExtractor (Bertin \& Arnouts 1996) and PSF fitting. In the FIR, we used efficient methods with strong background fluctuations: wavelet filtering, threshold detection and aperture photometry.

\subsubsection{Point spread function (PSF)}

The $24 \mu \mathrm{m}$ empirical PSF of each field is generated with the IRAF (image reduction and analysis facility ${ }^{2}$ ) DAOPHOT package (Stetson 1987) on the 30 brightest sources of each map. It is normalized in a 12 arcsec radius aperture. Aperture correction (1.19) is computed with the S Tiny $\operatorname{Tim}^{3}$ (Krist 2006) theoretical PSF for a constant $v S_{v}$ spectrum. The difference of correction between a $S_{v}=v^{-2}$ and a $v^{2}$ spectrum is less than $2 \%$. So, the hypothesis on the input spectrum is not critical for the PSF normalization.

At $70 \mu \mathrm{m}$ and $160 \mu \mathrm{m}$, we built a single empirical PSF from the SWIRE fields. We used the Starfinder PSF extraction routine (Diolaiti et al. 2000), which median-stacks the brightest non-saturated sources $\left(100 \mathrm{mJy}<S_{70}<10 \mathrm{Jy}\right.$ and $300 \mathrm{mJy}<S_{160}<1 \mathrm{Jy}$ ). Previously, fainter neighboring sources were subtracted with a first estimation of the PSF. At $70 \mu \mathrm{m}$ (resp. $160 \mu \mathrm{m}$ ), the normalization is done in a 35 arcsec (resp. 80 arcsec) aperture, with a sky annulus between 75 arcsec and 125 arcsec (resp. 150 arcsec and 250 arcsec); the aperture correction was 1.21 (resp. 1.20). The theoretical signal in the sky annulus and the aperture correction were computed with the $\mathrm{S}$ Tiny Tim Spitzer PSF for a constant $v S_{v}$ spectrum. These parameters do not vary more than $5 \%$ with the spectrum of sources. Pixels that were affected by the temporal median filtering artifact, which was sometimes present around bright sources, were masked prior to these operations.

\subsubsection{Source extraction and photometry}

At $24 \mu \mathrm{m}$, we detected sources with SExtractor. We chose a Gaussian filter (gauss_5.0_9x9.conv) and a background filter of the size of $64 \times 64$ pixels. The detection and analysis thresholds were tuned for each field. We performed PSF fitting photometry

\footnotetext{
2 http://iraf.noao.edu/

3 http://ssc.spitzer.caltech.edu/archanaly/ contributed/stinytim/
} 
with the DAOPHOT allstar routine. This routine is very efficient for blended sources flux measurement.

At $70 \mu \mathrm{m}$ and $160 \mu \mathrm{m}$, we applied the a-trou wavelet filtering (Starck et al. 1999) on the maps to remove the large scale fluctuations (10 pixels) on which we performed the source detection with a threshold algorithm (Dole et al. 2001, 2004). The threshold was tuned for each field. Photometry was done by aperture photometry on a non filtered map at the positions found on the wavelet filtered map. At $70 \mu \mathrm{m}$, we used 10 arcsec aperture radius and a 18 arcsec to 39 arcsec sky annulus. At $160 \mu \mathrm{m}$, we used an aperture of $20 \operatorname{arcsec}$ and a $40 \operatorname{arcsec}$ to $75 \operatorname{arcsec}$ annulus. Aperture corrections were computed with the normalized empirical PSF: 3.22 at $70 \mu \mathrm{m}$ and 3.60 at $160 \mu \mathrm{m}$. In order to estimate the uncertainty on this correction, aperture corrections were computed using five PSF built on five different SWIRE fields. The uncertainty is $1.5 \%$ at $70 \mu \mathrm{m}$ and $4.5 \%$ at $160 \mu \mathrm{m}$.

\subsection{Color correction}

The MIPS calibration factors were calculated for a $10000 \mathrm{~K}$ blackbody (MIPS Data Handbook 20074). However, the galaxies SED are different and the MIPS photometric bands are large $(\lambda / \Delta \lambda \approx 3)$. Thus, color corrections were needed. We used (like Shupe et al. (2008) and Frayer et al. (2009)) a constant $v S_{v}$ spectrum at $24 \mu \mathrm{m}, 70 \mu \mathrm{m}$ and $160 \mu \mathrm{m}$. Consequently, all fluxes were divided by $0.961,0.918$ and 0.959 at $24 \mu \mathrm{m}, 70 \mu \mathrm{m}$ and $160 \mu \mathrm{m}$ due to this color correction. Another possible convention is $v S_{v} \propto v^{-1}$. This convention is more relevant for the local sources at $160 \mu \mathrm{m}$, whose spectrum decreases quickly with wavelength. Nevertheless, the redshifted sources studied by stacking are seen at their peak of the cold dust emission, and their SED agrees better with the constant $v S_{v}$ convention. The difference of color correction between these two conventions is less than $2 \%$, and this choice is thus not critical. We consequently chose the constant $v S_{v}$ convention to more easily compare our results with Shupe et al. (2008) and Frayer et al. (2009).

\section{Catalog properties}

\subsection{Spurious sources}

Our statistical analysis may suffer from spurious sources. We have to estimate how many false detections are present in a map and what their flux distribution is. To do so, we built a cata$\log$ with the flipped map. To build this flipped map, we multiplied the values of the pixels of the original map by a factor of -1 . Detection and photometry parameters were exactly the same as for normal catalogs. At $24 \mu \mathrm{m}$, there are few spurious sources $(<10 \%)$ in bins brighter than the $80 \%$ completeness limit flux density. At $70 \mu \mathrm{m}$ and $160 \mu \mathrm{m}$, fluctuations of the background due to unresolved faint sources are responsible for spurious detections. Nevertheless, the ratio between detected source numbers and fake source numbers stayed reasonable (below 0.2) down to the $80 \%$ completeness limit (see the example of FIDEL CDFS at $70 \mu \mathrm{m}$ in Fig. 1).

\subsection{Completeness}

The completeness is the probability to extract a source of a given flux. To estimate it, we added artificial sources (based on empirical PSF) on the initial map and looked for a detection in a $2 \operatorname{arcsec}$ radius at $24 \mu \mathrm{m}$ around the initial position $(8 \operatorname{arcsec}$

${ }^{4}$ http://ssc.spitzer.caltech.edu/mips/dh/

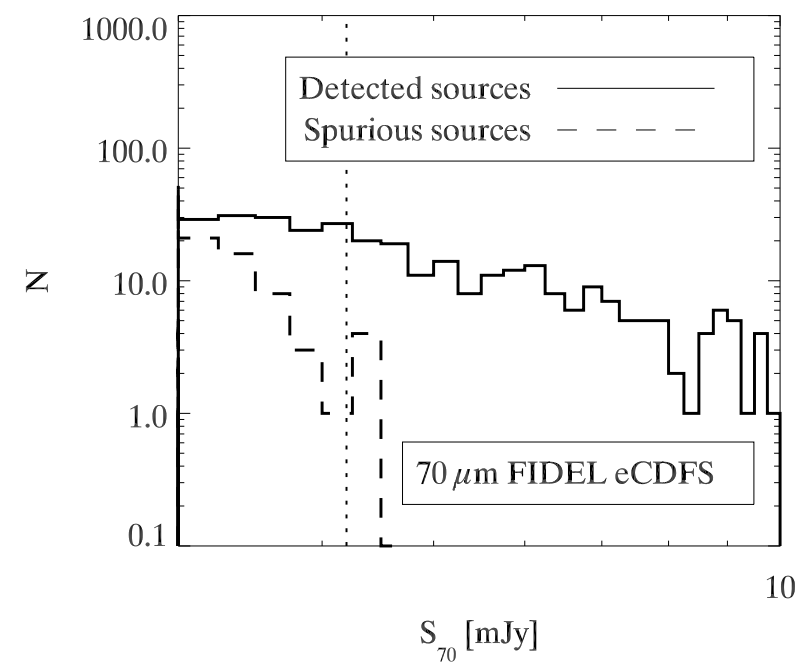

Fig. 1. Flux distribution of sources extracted from normal (solid line) and flipped (dash line) maps, at $70 \mu \mathrm{m}$ in FIDEL eCDFS. The vertical dashed line represents the $80 \%$ completeness flux density.

at $70 \mu \mathrm{m}$ and $16 \operatorname{arcsec}$ at $160 \mu \mathrm{m}$ ). This operation was done for different fluxes with a Monte-Carlo simulation. We chose the number of artificial sources in each realization in a way that they have less than $1 \%$ probability to fall at a distance shorter than 2 PSF FWHM (full width at half maximum). The completeness is plotted in Fig. 2, and the $80 \%$ completeness level is reported in Table 1.

\subsection{Photometric accuracy}

The photometric accuracy was checked with the same Monte-Carlo simulation. For different input fluxes, we built histograms of measured fluxes and computed the median and scatter of these distributions. At lower fluxes, fluxes are overestimated and errors are larger. These informations were used to estimate the Eddington bias (see next section). The photometric accuracy at $70 \mu \mathrm{m}$ in FIDEL CDFS is plotted as an example in Fig. 3.

We also compared our catalogs with published catalogs. At $24 \mu \mathrm{m}$, we compared it with the GOODS CDFS catalog of Chary et al. (2004), and the COSMOS catalog of LeFloc'h et al. (2009). Their fluxes were multiplied by a corrective factor to be compatible with the $v S_{v}=$ constant convention. Sources were considered to be the same if they are separated by less than 2 arcsec. We computed the standard deviation of the distribution of the ratio between our and their catalogs. In a 80-120 $\mu \mathrm{Jy}$ bin in the CDFS, we found a dispersion of $19 \%$. In a $150-250 \mu \mathrm{Jy}$ bin in COSMOS, we found a scatter of $13 \%$. The offset is $+3 \%$ with COSMOS catalog and $-1 \%$ with GOODS catalog. At 70 and $160 \mu \mathrm{m}$, we compared our catalogs with the COSMOS and SWIRE team ones. In all cases, the scatter is less than $15 \%$, and the offset is less than $3 \%$. At all wavelengths and for all fields, the offset is less than the calibration uncertainty.

\subsection{Eddington bias}

When sources become fainter, photometric errors increase. In addition, fainter sources are more numerous than brighter ones (in general $\mathrm{d} N / \mathrm{d} S \sim S^{-r}$ ). Consequently, the number of sources in faint bins are overestimated. This is the classical Eddington 


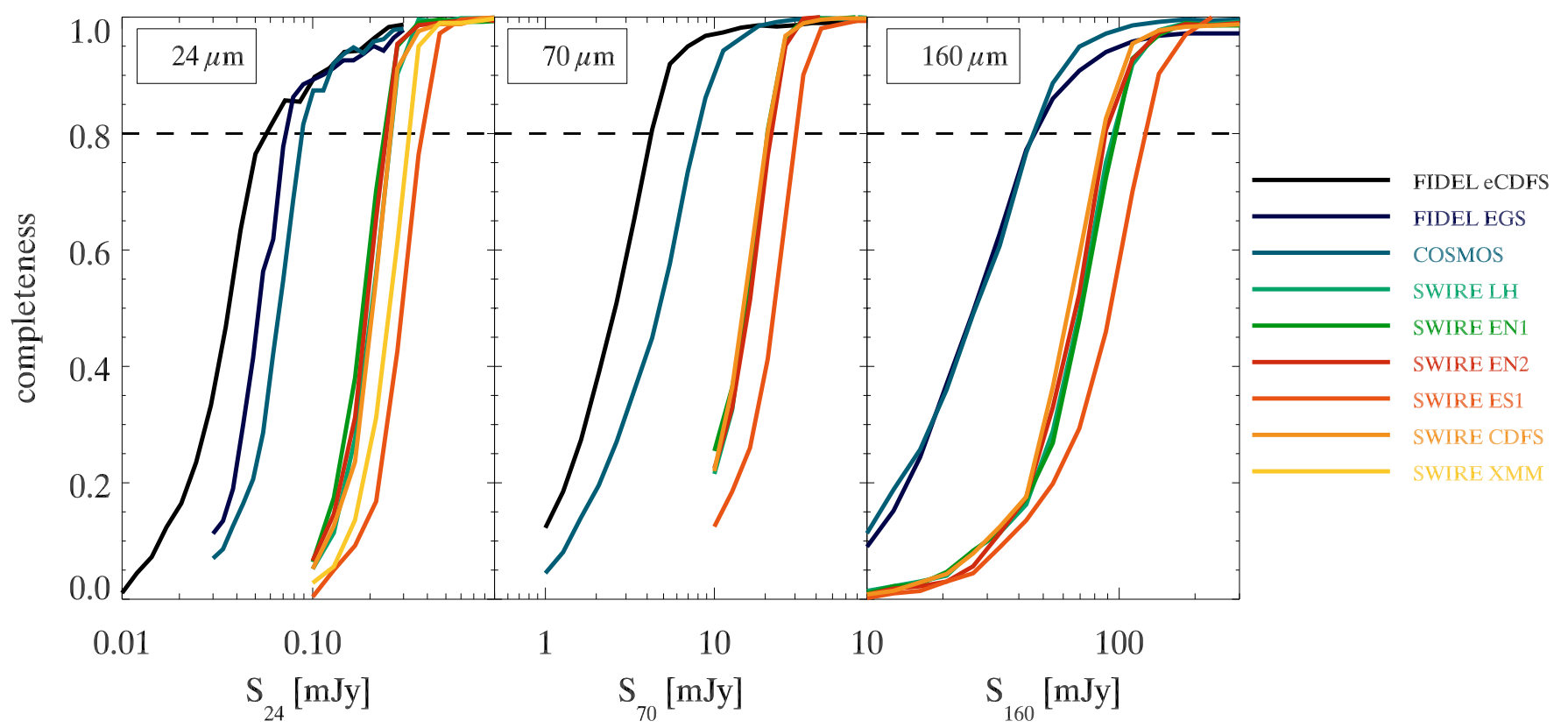

Fig. 2. Completeness at $24 \mu \mathrm{m}$ (left), $70 \mu \mathrm{m}$ (center), and $160 \mu \mathrm{m}$ (right) as a function of the source flux for all fields. The dashed line represents $80 \%$ completeness.

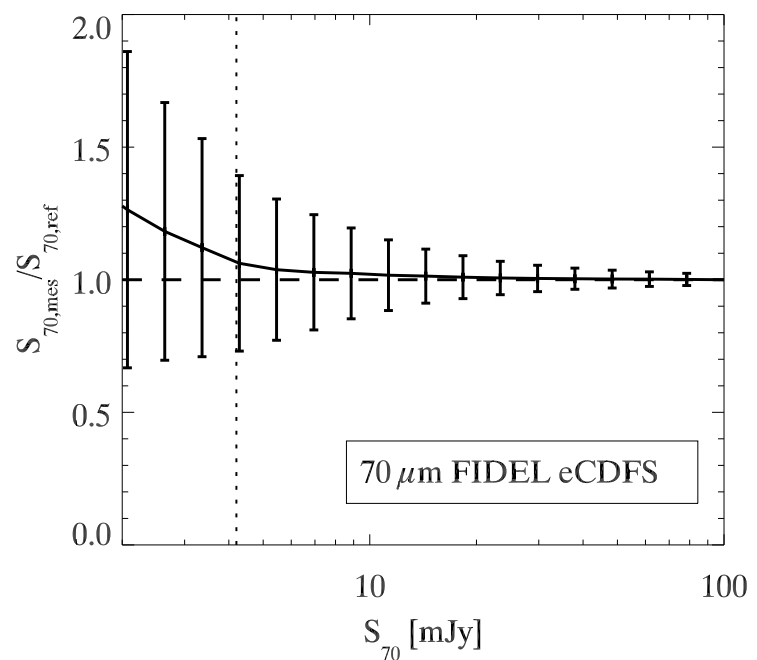

Fig. 3. Ratio between measured flux and input flux computed from Monte Carlo simulations at $70 \mu \mathrm{m}$ in FIDEL eCDFS. Error bars represent $1 \sigma$ dispersion. The vertical dashed line represents the $80 \%$ completeness flux density.

bias (Eddington 1913, 1940). The example of FIDEL CDFS at $70 \mu \mathrm{m}$ is plotted in Fig. 4.

To correct for this effect at $70 \mu \mathrm{m}$ and $160 \mu \mathrm{m}$, we estimated a correction factor for each flux bin. We generated an input flux catalog with a power-law distribution $(r=1.6$ at $70 \mu \mathrm{m}, r=3$ at $160 \mu \mathrm{m})$. We took into account completeness and photometric errors (coming from Monte-Carlo simulations) to generate a mock catalog. We then computed the ratio between the number of mock sources found in a bin and the number of input sources. This task was done for all fields. This correction is more important for large $r$ (at $160 \mu \mathrm{m}$ ). At $24 \mu \mathrm{m}$, thanks to the PSF fitting, the photometric error is more reduced and symmetrical. Less faint sources are thus placed in brighter flux bins. Because of this property and the low $r$ (about 1.45), this correction can be ignored for $24 \mu \mathrm{m}$ counts.

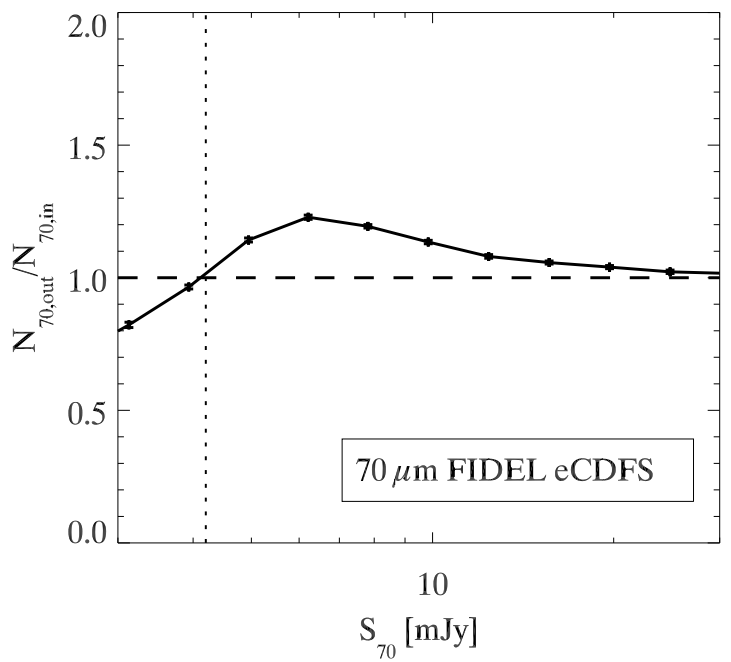

Fig. 4. Eddington bias: ratio between the number of detected sources and the number of input sources at $70 \mu \mathrm{m}$ in FIDEL eCDFS. The vertical dashed line represents the $80 \%$ completeness flux density.

\section{Number counts}

\subsection{Removing stars from the catalogs}

To compute extragalactic number counts at $24 \mu \mathrm{m}$, we removed the stars with the $K-[24]<2$ color criterion and identification procedure following Shupe et al. (2008). The $K$ band magnitudes were taken from the 2MASS catalog (Skrutskie et al. 2006). We ignored the star contribution at $70 \mu \mathrm{m}$ and $160 \mu \mathrm{m}$, which is negligible $(<1 \%$ in all used flux density bins) according to the DIRBE Faint Count model (Arendt et al. 1998).

\section{2. $24 \mu m$ number counts}

We counted the number of extragalactic sources for each field and in each flux bin. We subtracted the number of spurious detections (performed on the flipped map). We divided by the 


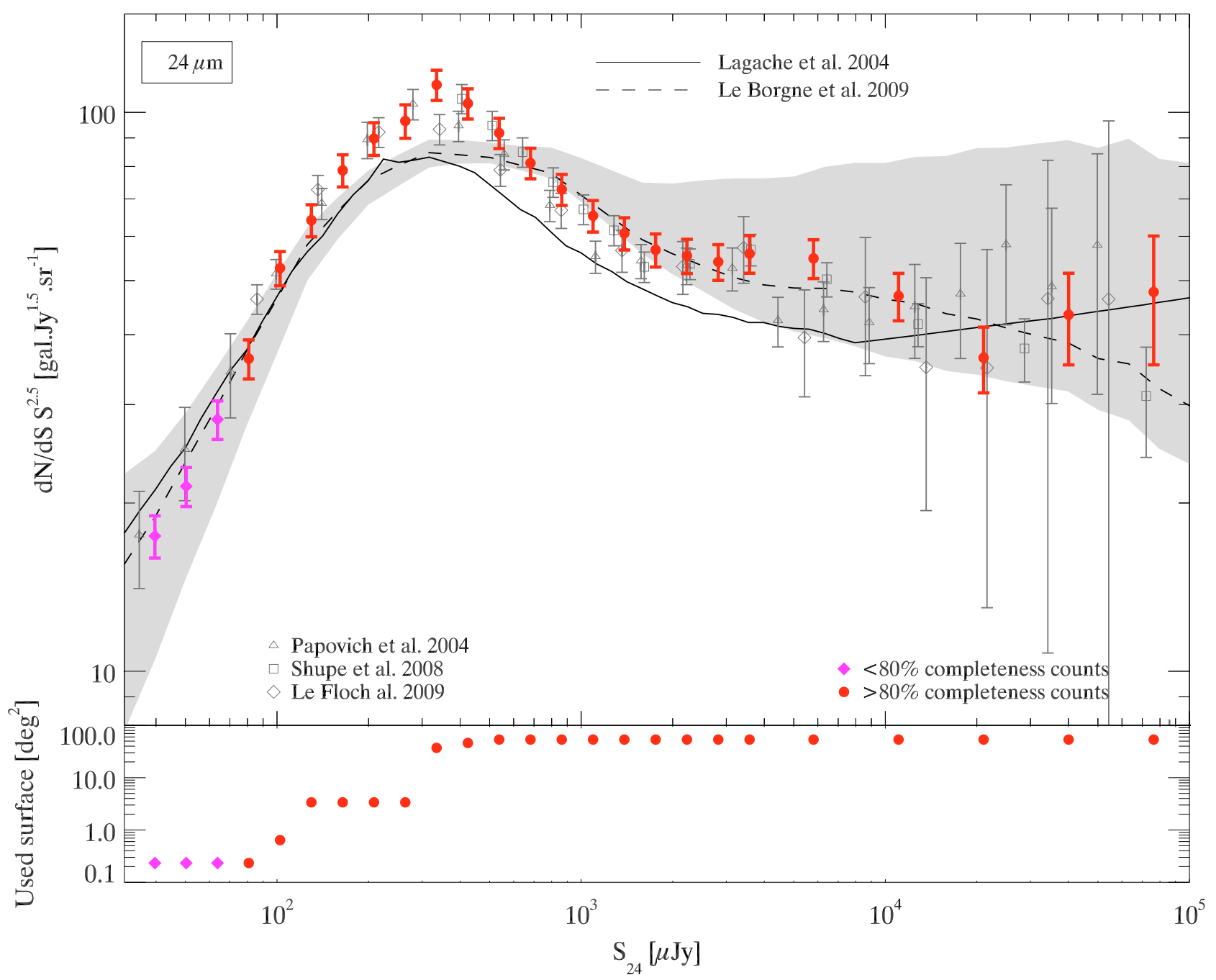

Fig. 5. Differential number counts at $24 \mu \mathrm{m}$. Filled circle: points obtained with $\geq 80 \%$ completeness; filled diamond: points obtained with a $50 \%$ to $80 \%$ completeness; open triangle: Papovich et al. (2004) GTO number counts obtained with PSF fitting photometry; open square: Shupe et al. (2008) SWIRE number counts obtained with aperture photometry; open diamond: LeFloc'h et al. (2009) COSMOS number counts obtained with PSF fitting photometry; continuous line: Lagache et al. (2004) model; dashed line and grey region: Le Borgne et al. (2009) model and 90\% confidence region. Error bars take into accounts clustering (see Sect. 4.5) and calibration uncertainties (Engelbracht et al. 2007).

completeness. As a next step, the counts of all fields were combined together with a mean weighted by field size. Actually, a weighting by the number of sources in each field overweighs the denser fields and biases the counts. Counts from a field were combined only if the lower end of the flux bin was larger then or equal to the $80 \%$ completeness. We thus reached $71 \mu \mathrm{Jy}(71 \mu \mathrm{Jy}$ to $90 \mu \mathrm{Jy}$ bin) in the counts. However, to probe fainter flux densities, we used the data from the deepest field (FIDEL eCDFS) between a 50 and $80 \%$ completeness, allowing us to reach $35 \mu \mathrm{Jy}$.

Our number counts are plotted in Fig. 5 and are written in Table 2. We also plot data from Papovich et al. (2004), Shupe et al. (2008) and LeFloc'h et al. (2009), and model predictions from Lagache et al. (2004) and Le Borgne et al. (2009). The Papovich et al. (2004) fluxes are multiplied by a factor 1.052 to take into account the update in the calibration, the color correction and the PSF. This correction of flux also implies a correction on number counts, according to:

$\left(\frac{\mathrm{d} N}{\mathrm{~d} S_{f}} S_{f}^{2.5}\right)_{S_{f}}=\left(c^{1.5} \frac{\mathrm{d} N}{\mathrm{~d} S_{i}} S_{i}^{2.5}\right)_{c S_{i}}$,

where $S_{i}$ is the initial flux, $S_{f}$ is the corrected flux and $c$ the corrective factor $\left(S_{f}=c S_{i}\right)$. A correction of the flux thus corresponds to a shift in the abscissa (factor $c$ ) and in the ordinate (factor $c^{1.5}$ ). Papovich et al. (2004) do not subtract stars and thus overestimate counts above $10 \mathrm{mJy}$. We have a very good agreement with their work below $10 \mathrm{mJy}$. We also have a very good agreement with Shupe et al. (2008). The LeFloc'h et al. (2009) fluxes are multiplied by 1.05 to take into account a difference of the reference SED: $10000 \mathrm{~K}$ versus constant $v S_{v}$, and by another correction of $3 \%$ corresponding to the offset observed in Sect. 3.3. There is an excellent agreement with their work.

The Lagache et al. $(2004)^{5}$ and Le Borgne et al. (2009) ${ }^{6}$ generally agree well with the data, in particular on the faint end below $100 \mu \mathrm{Jy}$, and on the position of the peak around $300 \mu \mathrm{Jy}$. However, the Lagache et al. (2004) model slightly underestimates (about 10\%) the counts above $200 \mu \mathrm{Jy}$. The Le Borgne et al. (2009) model is flatter than the data, and agrees reasonably well above $600 \mu \mathrm{Jy}$.

5 Lagache et al. (2004) model used a $\Lambda \mathrm{CDM}$ cosmology with $\Omega_{\Lambda}=$ $0.73, \Omega_{\mathrm{M}}=0.27$ and $h=0.71$.

${ }^{6}$ Le Borgne et al. (2009) model used a $\Lambda$ CDM cosmology with $\Omega_{\Lambda}=$ $0.7, \Omega_{\mathrm{M}}=0.3$ and $h=0.7$. 
Table 2. Differential number counts at $24 \mu \mathrm{m}$.

\begin{tabular}{rrrrrrrr}
\hline \hline$\langle S\rangle$ & \multicolumn{1}{c}{$S_{\min }$} & $S_{\max }$ & $\mathrm{d} N / \mathrm{d} S . S^{2.5}$ & $\sigma_{\text {poisson }}$ & $\sigma_{\text {clustering }}$ & $\sigma_{\text {clus. calib. }}$ & $\Omega_{\text {used }}$ \\
\hline & (in mJy) & & & (in gal Jy $\mathrm{deg}^{1.5} \mathrm{sr}^{-1}$ ) & & $\mathrm{deg}^{2}$ \\
0.040 & 0.035 & 0.044 & 17.5 & 1.0 & 1.1 & 1.3 & 0.2 \\
0.050 & 0.044 & 0.056 & 21.4 & 1.0 & 1.1 & 1.4 & 0.2 \\
0.064 & 0.056 & 0.071 & 28.2 & 1.2 & 1.5 & 1.8 & 0.2 \\
0.081 & 0.071 & 0.090 & 36.2 & 1.5 & 1.9 & 2.4 & 0.2 \\
0.102 & 0.090 & 0.114 & 52.6 & 1.3 & 1.9 & 2.9 & 0.6 \\
0.130 & 0.114 & 0.145 & 64.1 & 1.0 & 1.7 & 3.1 & 3.4 \\
0.164 & 0.145 & 0.184 & 78.7 & 1.1 & 2.2 & 3.8 & 3.4 \\
0.208 & 0.184 & 0.233 & 89.8 & 1.3 & 2.8 & 4.5 & 3.4 \\
0.264 & 0.233 & 0.295 & 96.5 & 1.5 & 3.3 & 5.1 & 3.4 \\
0.335 & 0.295 & 0.374 & 112.0 & 0.8 & 1.8 & 4.8 & 37.2 \\
0.424 & 0.374 & 0.474 & 103.7 & 0.6 & 1.7 & 4.5 & 46.1 \\
0.538 & 0.474 & 0.601 & 91.9 & 0.6 & 1.5 & 4.0 & 53.6 \\
0.681 & 0.601 & 0.762 & 81.2 & 0.6 & 1.5 & 3.6 & 53.6 \\
0.863 & 0.762 & 0.965 & 72.8 & 0.7 & 1.6 & 3.3 & 53.6 \\
1.094 & 0.965 & 1.223 & 65.3 & 0.8 & 1.6 & 3.1 & 53.6 \\
1.387 & 1.223 & 1.550 & 60.8 & 0.9 & 1.7 & 3.0 & 53.6 \\
1.758 & 1.550 & 1.965 & 56.7 & 1.0 & 1.8 & 2.9 & 53.6 \\
2.228 & 1.965 & 2.490 & 55.4 & 1.2 & 2.1 & 3.0 & 53.6 \\
2.823 & 2.490 & 3.156 & 54.0 & 1.5 & 2.3 & 3.2 & 53.6 \\
3.578 & 3.156 & 4.000 & 55.9 & 1.8 & 2.7 & 3.5 & 53.6 \\
5.807 & 4.000 & 7.615 & 54.8 & 1.5 & 2.9 & 3.6 & 53.6 \\
11.055 & 7.615 & 14.496 & 46.9 & 2.3 & 3.6 & 4.1 & 53.6 \\
21.045 & 14.496 & 27.595 & 36.4 & 3.3 & 4.4 & 4.6 & 53.6 \\
40.063 & 27.595 & 52.531 & 43.4 & 5.9 & 7.7 & 7.9 & 53.6 \\
76.265 & 52.531 & 100.000 & 47.7 & 9.9 & 12.0 & 12.2 & 53.6 \\
\hline
\end{tabular}

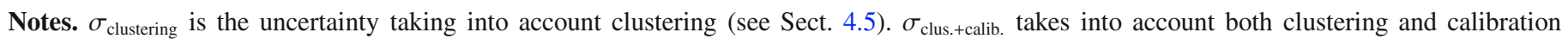
(Engelbracht et al. 2007).

Table 3. Differential number counts at $70 \mu \mathrm{m}$.

\begin{tabular}{rrrrrrrr}
\hline \hline$\langle S\rangle$ & $S_{\min }$ & $S_{\max }$ & $\mathrm{d} N / \mathrm{d} S . S^{2.5}$ & $\sigma_{\text {poisson }}$ & $\sigma_{\text {clustering }}$ & $\sigma_{\text {clus.+calib. }}$ & $\Omega_{\text {used }}$ \\
\hline & (in mJy) & & & \multicolumn{2}{c}{ (in gal Jy $\left.\mathrm{de}^{1.5} \mathrm{Sr}^{-1}\right)$} & & $\mathrm{deg}^{2}$ \\
4.197 & 3.500 & 4.894 & 2073. & 264. & 309. & 342. & 0.2 \\
5.868 & 4.894 & 6.843 & 2015. & 249. & 298. & 330. & 0.2 \\
8.206 & 6.843 & 9.569 & 1690. & 289. & 332. & 353. & 0.2 \\
11.474 & 9.569 & 13.380 & 2105. & 123. & 202. & 250. & 2.6 \\
16.044 & 13.380 & 18.708 & 2351. & 148. & 228. & 281. & 2.6 \\
22.434 & 18.708 & 26.159 & 1706. & 153. & 208. & 240. & 2.6 \\
31.369 & 26.159 & 36.578 & 2557. & 69. & 124. & 218. & 38.1 \\
43.862 & 36.578 & 51.146 & 2446. & 73. & 123. & 211. & 45.5 \\
61.331 & 51.146 & 71.517 & 2359. & 90. & 141. & 217. & 45.5 \\
85.758 & 71.517 & 100.000 & 2257. & 112. & 164. & 228. & 45.5 \\
157.720 & 100.000 & 215.440 & 2354. & 121. & 198. & 257. & 45.5 \\
339.800 & 215.440 & 464.160 & 2048. & 200. & 276. & 311. & 45.5 \\
732.080 & 464.160 & 1000.000 & 2349. & 381. & 500. & 526. & 45.5 \\
\hline
\end{tabular}

Notes. $\sigma_{\text {clustering }}$ is the uncertainty taking into account clustering (see Sect. 4.5). $\sigma_{\text {clus.tcalib. }}$ takes into account both clustering and calibration (Gordon et al. 2007).

\section{3. $70 \mu \mathrm{m}$ number counts}

Counts in the flux density bins brighter than the $80 \%$ completeness limit were obtained in the same way as at $24 \mu \mathrm{m}$ (Fig. 6 and Table 3). In addition, they were corrected from the Eddington bias (cf. Sect. 3.4). We reached about 4.9 mJy at $80 \%$ completeness (4.9 to 6.8 bin). We used CDFS below $80 \%$ completeness limit to probe fainter flux density level. We cut these counts at $3.5 \mathrm{mJy}$. At this flux density, the spurious rate reached $50 \%$. We used a stacking analysis to probe fainter flux density levels (cf. Sect. 5).
We can see breaks in the counts around $10 \mathrm{mJy}$ and $20 \mathrm{mJy}$. These breaks appear between points built with a different set of fields. Our counts agree with earlier works of Dole et al. (2004), Frayer et al. (2006) and Frayer et al. (2009). However, these works suppose only a Poissonian uncertainty, which underestimates the error bars (see Sect. 4.5). Our data also agree well with these works. The Lagache et al. (2004) model agrees well with our data. The Le Borgne et al. (2009) model gives a reasonable fit, despite an excess of about $30 \%$ between $3 \mathrm{mJy}$ and $10 \mathrm{mJy}$. 
M. Béthermin et al.: Spitzer deep wide legacy MIR/FIR counts and CIB lower limits

Table 4. Differential number counts at $160 \mu \mathrm{m} . \sigma_{\text {clustering }}$ is the uncertainty taking into account clustering (see Sect. 4.5).

\begin{tabular}{rrrrrrrr}
\hline \hline$\langle S\rangle$ & $S_{\min }$ & $S_{\max }$ & $\mathrm{d} N / \mathrm{d} S . S^{2.5}$ & $\sigma_{\text {poisson }}$ & $\sigma_{\text {clustering }}$ & $\sigma_{\text {clus. +calib. }}$ & $\Omega_{\text {used }}$ \\
\hline & (in $\mathrm{mJy})$ & & & \multicolumn{2}{c}{ (in gal Jy ${ }^{1.5} \mathrm{sr}^{-1}$ ) } & & $\mathrm{deg}^{2}$ \\
45.747 & 40.000 & 51.493 & 16855. & 1312. & 2879. & 3519. & 3.0 \\
58.891 & 51.493 & 66.289 & 14926. & 1243. & 2704. & 3243. & 3.0 \\
75.813 & 66.289 & 85.336 & 13498. & 1319. & 2648. & 3104. & 3.0 \\
97.596 & 85.336 & 109.860 & 12000. & 1407. & 2442. & 2835. & 3.0 \\
125.640 & 109.860 & 141.420 & 10687. & 457. & 991. & 1621. & 36.2 \\
161.740 & 141.420 & 182.060 & 7769. & 425. & 773. & 1211. & 42.9 \\
208.210 & 182.060 & 234.370 & 7197. & 472. & 810. & 1184. & 42.9 \\
268.040 & 234.370 & 301.710 & 5406. & 487. & 734. & 979. & 42.9 \\
345.050 & 301.710 & 388.400 & 5397. & 585. & 843. & 1063. & 42.9 \\
444.200 & 388.400 & 500.000 & 4759. & 662. & 891. & 1059. & 42.9 \\
750.000 & 500.000 & 1000.000 & 6258. & 685. & 1158. & 1380. & 42.9 \\
1500.000 & 1000.000 & 2000.000 & 4632. & 989. & 1379. & 1487. & 42.9 \\
\hline
\end{tabular}

Notes. $\sigma_{\text {clus.tcalib. }}$ takes into account both clustering and calibration (Stansberry et al. 2007).

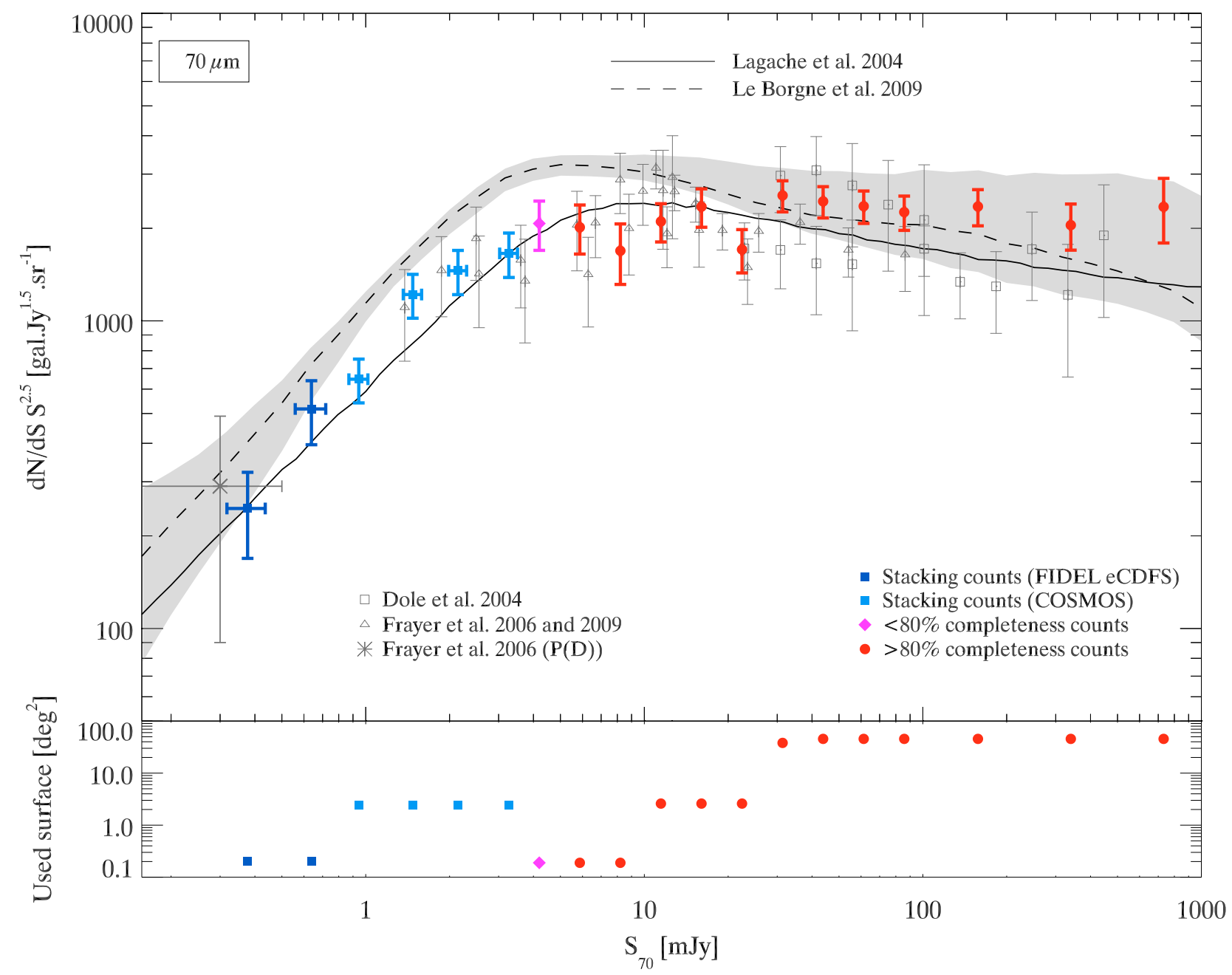

Fig. 6. Differential number counts at $70 \mu \mathrm{m}$. Filled circle: points obtained with $\geq 80 \%$ completeness; filled diamond: points obtained with less than 50\% spurious sources and less than 80\% completeness; filled square: stacking number counts (clear: FIDEL eCDFS, dark: COSMOS); open square: Dole et al. (2004) number counts in CDFS, Bootes and Marano; open triangle: Frayer et al. (2006) in GOODS and Frayer et al. (2009) in COSMOS; cross: Frayer et al. (2006) deduced from background fluctuations; continuous line: Lagache et al. (2004) model; dashed line and grey region: Le Borgne et al. (2009) model and 90\% confidence region. Error bars take into account clustering (see Sect. 4.5) and calibration uncertainties (Gordon et al. 2007).

\section{4. $160 \mu m$ number counts}

The $160 \mu \mathrm{m}$ number counts were obtained exactly in the same way as at $70 \mu \mathrm{m}$. We used COSMOS and EGS to probe counts below the $80 \%$ completeness limit. We reached $51 \mathrm{mJy}$ at $80 \%$ completeness (51 mJy to $66 \mathrm{mJy}$ bin) and $40 \mathrm{mJy}$ for the $50 \%$ spurious rate cut (Fig. 7 and Table 4). We used a stacking analysis to probe fainter flux density levels (cf. Sect. 5). 


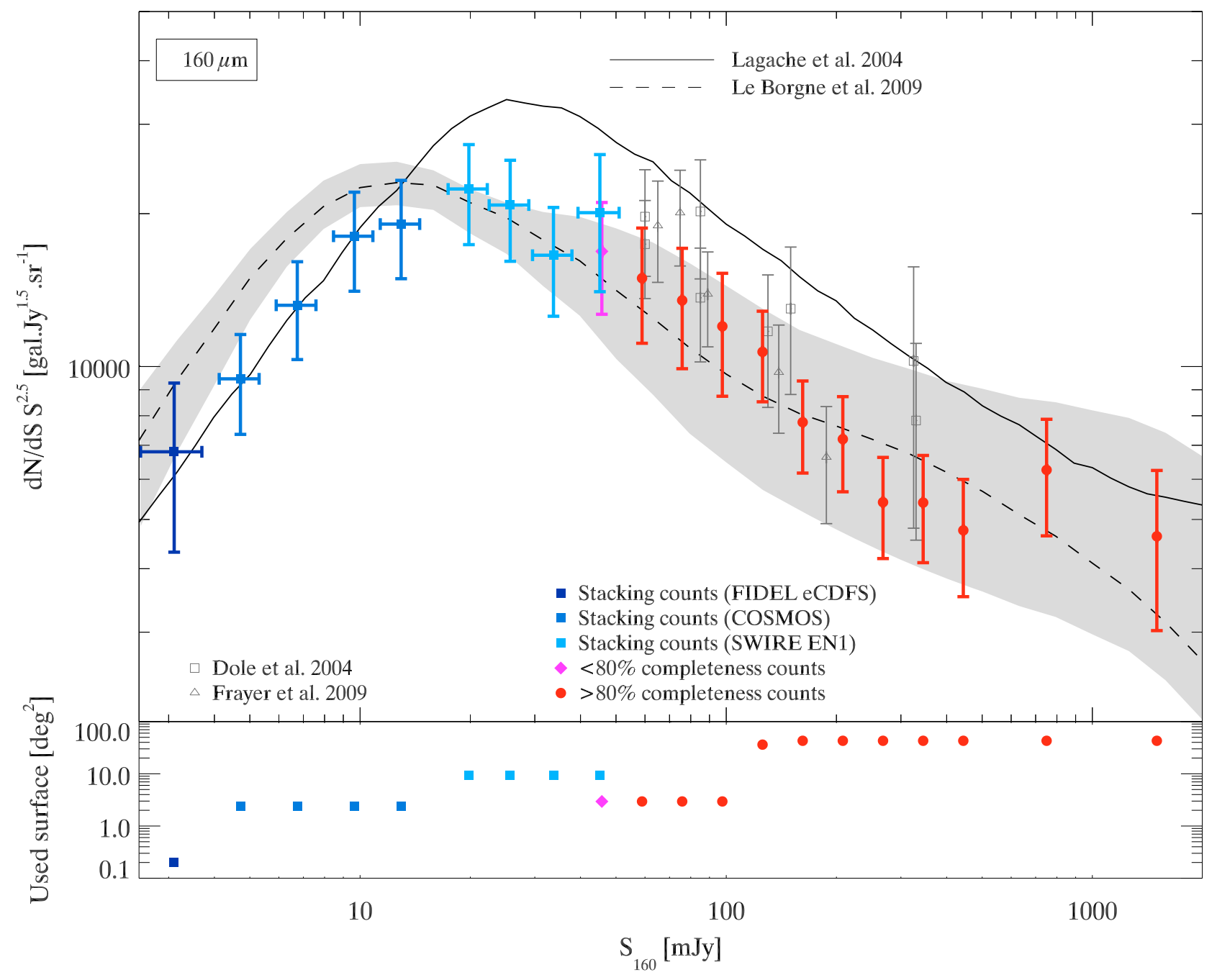

Fig. 7. Differential number counts at $160 \mu \mathrm{m}$. Filled circle: points obtained with $\geq 80 \%$ completeness; filled diamond: points obtained with less than $50 \%$ spurious sources and less than $80 \%$ completeness; filled square: stacking number counts (clear: FIDEL/GTO CDFS, middle: COSMOS, dark: SWIRE EN1); open square: Dole et al. (2004) number counts in CDFS and Marano; open triangle: Frayer et al. (2009) in COSMOS; continuous line: Lagache et al. (2004) model; dashed line and grey region: Le Borgne et al. (2009) model and 90\% confidence region. Error bars take into account clustering (see Sect. 4.5) and calibration uncertainties (Stansberry et al. 2007).

Our counts agree with the earlier works of Dole et al. (2004) and Frayer et al. (2009). We find like Frayer et al. (2009) that the Lagache et al. (2004) model overestimates the counts by about $30 \%$ above $50 \mathrm{mJy}$ (see the discussion in Sect. 7.2). On the contrary, the Le Borgne et al. (2009) model underpredicts the counts by about $20 \%$ between $50 \mathrm{mJy}$ and $150 \mathrm{mJy}$.

\subsection{Uncertainties on number counts including clustering}

Shupe et al. (2008) showed that the SWIRE field-to-field variance is significantly higher than the Poisson noise (by a factor of three in some flux bins). They estimated their uncertainties on number counts with a field bootstrap method. We used a more formal method to deal with this problem.

The uncertainties on the number counts are Poissonian only if sources are distributed uniformly. But, actually, the infrared galaxies are clustered. The uncertainties must thus be computed taking into account clustering. We first measured the source clustering as a function of the flux density with the counts-in-cells moments (c-in-c) method (Peebles 1980; Szapudi 1998; Blake \& Wall 2002). We then computed the uncertainties knowing these clustering properties of the sources, the source density in the flux density bins and the field shapes. The details are explained in the Appendix A.

This statistical uncertainty can be combined with the Spitzer calibration uncertainty (Engelbracht et al. 2007; Gordon et al. 2007; Stansberry et al. 2007) to compute the total uncertainty on differential number counts.

\section{Deeper FIR number counts using a stacking analysis}

\subsection{Method}

The number counts derived in Sect. 4 show that down to the $80 \%$ completeness limit, the source surface density is $24100 \mathrm{deg}^{-2}$, $1200 \mathrm{deg}^{-2}$, and $220 \mathrm{deg}^{-2}$ at 24,70 , and $160 \mu \mathrm{m}$, respectively, i.e. 20 times (resp. 110 times) higher at $24 \mu \mathrm{m}$ than at $70 \mu \mathrm{m}$ (resp. $160 \mu \mathrm{m}$ ). These differences can be explained by the angular resolution decreasing with increasing wavelength, thus increasing confusion, and the noise properties of the detectors. There are thus many $24 \mu \mathrm{m}$ sources without detected FIR 
counterparts. If we want to probe deeper into the FIR number counts, we can take advantage of the information provided by the $24 \mu \mathrm{m}$ data, namely the existence of infrared galaxies not necessarily detected in the FIR, and their positions.

We used a stacking analysis (Dole et al. 2006) to determine the FIR/MIR color as a function of the MIR flux. With this information, we can convert MIR counts into FIR counts. The stacking technique consists in piling up very faint far-infrared galaxies which are not detected individually, but are detected at $24 \mu \mathrm{m}$. For this purpose, it makes use of the $24 \mu \mathrm{m}$ data prior to tracking their undetected counterpart at $70 \mu \mathrm{m}$ and $160 \mu \mathrm{m}$, where most of the bolometric luminosity arises. This method was used by Dole et al. (2006), who managed to resolve the FIR CIB using $24 \mu \mathrm{m}$ sources positions, as well many other authors (e.g Serjeant et al. (2004); Dye et al. (2006); Wang et al. (2006); Devlin et al. (2009); Dye et al. (2009); Marsden et al. (2009); Pascale et al. (2009)).

To derive the $70 \mu \mathrm{m}$ or $160 \mu \mathrm{m}$ versus $24 \mu \mathrm{m}$ color, we stacked the FIR maps (cleaned of bright sources) at the positions of the $24 \mu \mathrm{m}$ sources sorted by flux, and performed aperture photometry (same parameters as in Sect. 2). We thus get

$\overline{S_{\mathrm{FIR}}}=f\left(\overline{S_{24}}\right)$,

where $\overline{S_{\text {FIR }}}$ is the average flux density in the FIR of the population selected at $24 \mu \mathrm{m}, \overline{S_{24}}$ the average flux density at $24 \mathrm{mi}-$ crons, and $\mathrm{f}$ is the function linking both quantities. We derive f empirically using the $S_{\text {FIR }}$ versus $S_{24}$ relation obtained from stacking.

We checked that $f$ is a smooth monotonic function, in agreement with the expectation that the color varies smoothly with the redshift and the galaxy emission properties. Assuming that the individual sources follow this relation exactly, the FIR number counts could be deduced from

$$
\left.\frac{\mathrm{d} N}{\mathrm{~d} S_{\mathrm{FIR}}}\right|_{S_{\mathrm{FIR}}=f\left(S_{24}\right)}=\left.\frac{\mathrm{d} N}{\mathrm{~d} S_{24}}\right|_{S_{24}} /\left.\frac{\mathrm{d} S_{\mathrm{FIR}}}{\mathrm{d} S_{24}}\right|_{S_{24}} .
$$

In practice, the two first terms are discrete. In addition, the last term is computed numerically in the same $S_{24}$ bin, using the two neighboring flux density bins $(k-1$ and $k+1)$. We finally get

$\frac{\mathrm{d} N}{\mathrm{~d} S_{\mathrm{FIR}}}\left(\overline{S_{\mathrm{FIR}}}\right)=\left(\frac{\mathrm{d} N}{\mathrm{~d} S_{24}}\right)_{k} / \frac{\overline{S_{\mathrm{FIR}, k+1}}-\overline{S_{\mathrm{FIR}, k-1}}}{\overline{S_{24, k+1}}-\overline{S_{24, k-1}}}$,

where $\left\langle S_{\mathrm{FIR}}\right\rangle$ is measured by stacking. In reality, sources do not follow Eq. (2) exactly, but exhibit a scatter around this mean relation

$S_{\mathrm{FIR}}=f\left(S_{24}\right)+\sigma$.

Our method is still valid under the condition $\frac{\sigma}{f} \ll 1$, and we verify its validity with simulations (see next section).

To obtain a better signal to noise ratio, we cleaned the resolved bright sources from the FIR maps prior to stacking. We used $8 S_{24}$ bins per decade. We stacked a source only if the coverage was more than half of the median coverage of the map. Uncertainties on the FIR mean flux were estimated with a bootstrap method. Furthermore, knowing the uncertainties on the $24 \mu \mathrm{m}$ number counts and the mean $S_{24}$ fluxes, we deduced the uncertainties on the FIR number counts according to Eq. (4).

At $70 \mu \mathrm{m}$, we used the FIDEL eCDFS (cleaned at $S_{70}>$ $10 \mathrm{mJy}$ ) and the COSMOS (cleaned at $S_{70}>50 \mathrm{mJy}$ ) fields. At $160 \mu \mathrm{m}$, we used $160 \mu \mathrm{m}$ the GTO CDFS (cleaned at $S_{160}>$ $60 \mathrm{mJy}$ ), the COSMOS (cleaned at $S_{160}>100 \mathrm{mJy}$ ) and the SWIRE EN1 (no clean to probe the $S_{160}>20$ mJy sources) fields.

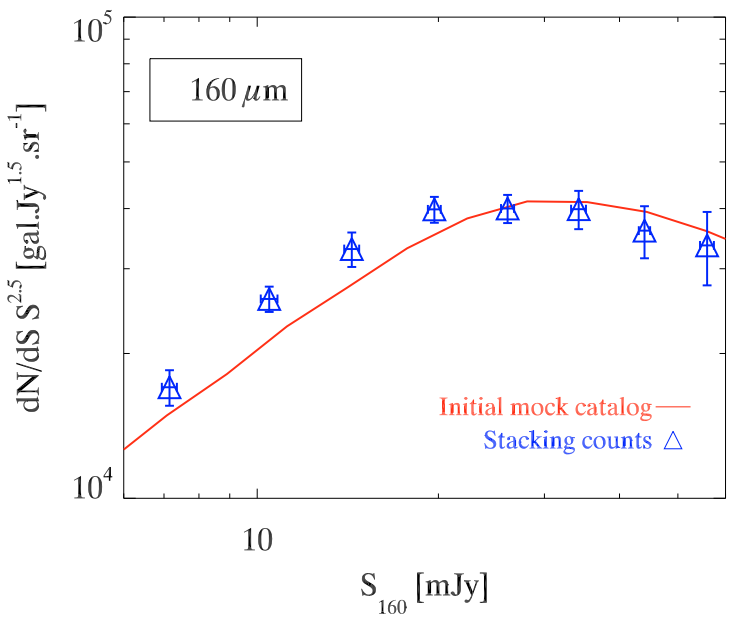

Fig. 8. Simulated number counts at $160 \mu \mathrm{m}$, computed from stacking counts (triangle) and from the input mock catalog (solid line). The good agreement (better than 15\%) validates the stacking counts method (see Sect. 5.2). Twenty realizations of the $2.9 \mathrm{deg}^{2}$ field maps with about 870000 mock sources each were used.

\subsection{Validation on simulations}

We used the Fernandez-Conde et al. (2008) simulations ${ }^{7}$ to validate our method. These simulations are based on the Lagache et al. (2004) model, and include galaxy clustering. We employed 20 simulated mock catalogs of a $2.9 \mathrm{deg}^{2}$ field each, containing about 870000 mock sources each. The simulated maps have the same pixel size as the actual Spitzer mosaics $\left(1.2^{\prime \prime}\right.$, $4^{\prime \prime}$, and $8^{\prime \prime}$ at $24 \mu \mathrm{m}, 70 \mu \mathrm{m}$, and $160 \mu \mathrm{m}$, resp.) and are convolved with our empirical PSF. A constant standard deviation Gaussian noise was added. We applied the same method as for the real data to produce stacking number counts. At $160 \mu \mathrm{m}$, for bins below $15 \mathrm{mJy}$, we cleaned the sources brighter than $50 \mathrm{mJy}$. Figure 8 shows $160 \mu$ m number counts from the mock catalogs down to $S_{160}=1 \mathrm{mJy}$ (diamond) and the number counts deduced from the stacking analysis described in the previous section (triangle). The error bars on the figure are the standard deviations of the 20 realization. There is a good agreement between the stacking counts and the classical counts (better than 15\%). Nevertheless, we observed a systematic bias, intrinsic to the method, of about $10 \%$ in some flux density bins. We thus combined this $10 \%$ error with the statistical uncertainties to compute our error bars. We also validated the estimation of the statistical uncertainty in the stacking counts: we check that the dispersion of the counts obtained by stacking, coming from different realizations, was compatible with our estimation of statistical uncertainties. The results are the same at $70 \mu \mathrm{m}$.

\subsection{Results}

At $70 \mu \mathrm{m}$, the stacking number counts reach $0.38 \mathrm{mJy}$ (see Fig. 6 and Table 5). The last stacking point is compatible with the Frayer et al. (2006) P(D) constraint. The stacking points also agree very well with the Lagache et al. (2004) model. The Le Borgne et al. (2009) model predicts slightly too many sources in 0.3 to $3 \mathrm{mJy}$ range. The turnover around $3 \mathrm{mJy}$ and the power law behavior of the faint counts $\left(S_{70}<2 \mathrm{mJy}\right)$, observed by Frayer et al. (2006) are confirmed with a better accuracy.

At $160 \mu \mathrm{m}$, the stacking counts reach $3.1 \mathrm{mJy}$ (see Fig. 7 and Table 6). We observed for the first time a turnover at about

7 Publicly available at http : //www . ias.u-psud.fr/irgalaxies/ 
Table 5. Stacking extragalactic number counts at $70 \mu \mathrm{m}$.

\begin{tabular}{lrrrr}
\hline \hline$\langle S\rangle$ & $\mathrm{d} N / \mathrm{d} S . S^{2.5}$ & $\sigma_{\text {clus. }} \sigma_{\text {clus. }+ \text { calib. }}$ & & \multirow{2}{*}{ Field } \\
\cline { 1 - 4 } (in mJy) & \multicolumn{3}{c}{ (in gal Jy ${ }^{1.5} \mathrm{sr}^{-1}$ ) } & \\
\hline $0.38 \pm 0.05$ & 246. & 72. & 76. & FIDEL CDFS \\
$0.64 \pm 0.07$ & 517. & 109. & 122. & FIDEL CDFS \\
$0.94 \pm 0.03$ & 646. & 80. & 105. & COSMOS \\
$1.48 \pm 0.04$ & 1218. & 151. & 198. & COSMOS \\
$2.14 \pm 0.05$ & 1456. & 183. & 239. & COSMOS \\
$3.27 \pm 0.07$ & 1657. & 211. & 273. & COSMOS \\
\hline
\end{tabular}

Notes. $\sigma_{\text {clus. }}$ is the uncertainty taking into account clustering (see Sect. 4.5). $\sigma_{\text {clus.tcalib. }}$ takes into account both clustering and calibration (Gordon et al. 2007).

Table 6. Stacking extragalactic number counts at $160 \mu \mathrm{m}$.

\begin{tabular}{lrrrr}
\hline \hline$\langle S\rangle$ & $\mathrm{d} N / \mathrm{d} S . S^{2.5}$ & $\sigma_{\text {clus. }} \sigma_{\text {clus.+calib. }}$ & \multirow{2}{*}{ Field } \\
\cline { 1 - 4 } (in mJy) & \multicolumn{3}{c}{ (in gal Jy $\left.{ }^{1.5} \mathrm{sr}^{-1}\right)$} & \\
\hline $3.11 \pm 0.46$ & 6795. & 2163. & 2485. & GTO CDFS \\
$4.71 \pm 0.16$ & 9458. & 1236. & 2104. & COSMOS \\
$6.74 \pm 0.22$ & 13203. & 1627. & 2880. & COSMOS \\
$9.65 \pm 0.26$ & 18057. & 2307. & 3986. & COSMOS \\
$12.95 \pm 0.37$ & 19075. & 2388. & 4182. & COSMOS \\
$19.82 \pm 0.48$ & 22366. & 2944. & 4987. & SWIRE EN1 \\
$25.71 \pm 0.81$ & 20798. & 2811. & 4682. & SWIRE EN1 \\
$33.74 \pm 0.98$ & 16567. & 2671. & 4004. & SWIRE EN1 \\
$45.18 \pm 2.08$ & 20089. & 4849. & 6049. & SWIRE EN1 \\
\hline
\end{tabular}

Notes. $\sigma_{\text {clus. }}$ is the uncertainty taking into account clustering (see

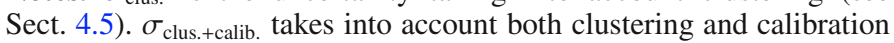
(Stansberry et al. 2007).

$20 \mathrm{mJy}$, and a power-law decrease at smaller flux densities. The stacking counts are lower than the Lagache et al. (2004) model around $20 \mathrm{mJy}$ (about 30\%). Below $15 \mathrm{mJy}$, the stacking counts agree with this model. The Le Borgne et al. (2009) model agree quite well with our points below $20 \mathrm{mJy}$. The results at $160 \mu \mathrm{m}$ will be discussed in Sect. 7.2.

\section{New lower limits and estimates of the CIB at $24 \mu \mathrm{m}, 70 \mu \mathrm{m}$ and $160 \mu \mathrm{m}$}

\section{1. $24 \mu \mathrm{m}$ CIB: lower limit and estimate}

By integrating the measured $24 \mu \mathrm{m}$ number counts between $35 \mu \mathrm{Jy}$ and $0.1 \mathrm{Jy}$, we can estimate a lower value of the CIB at this wavelength. The counts were integrated with a trapeze method. We estimated the uncertainty on the integral by adding (on 10000 realisations) a random Gaussian error to each data point with the $\sigma$ given by the count uncertainties taking into account clustering. We then added the 4\% calibration error of the instrument (Engelbracht et al. 2007). We found $2.26_{-0.09}^{+0.09} \mathrm{nW} \mathrm{m}^{-2} \mathrm{sr}^{-1}$. The very bright source counts $\left(S_{24}>\right.$ $0.1 \mathrm{Jy})$ are supposed to be euclidian $\left(\mathrm{d} N / \mathrm{d} S=C_{\text {eucl }} S^{2.5}\right)$. We used the three brightest points to estimate $C_{\text {eucl }}$. We found a contribution to the CIB of $0.032_{-0.003}^{+0.003} \mathrm{nW} \mathrm{m}^{-2} \mathrm{sr}^{-1}$. Consequently, very bright sources extrapolation is not critical for the CIB estimation (1\% of CIB). The contribution of $S_{24}>35 \mu \mathrm{Jy}$ is thus $2.29_{-0.09}^{+0.09} \mathrm{nW} \mathrm{m}^{-2} \mathrm{sr}^{-1}$ (cf. Table 7).

We might have wanted to estimate the CIB value at $24 \mu \mathrm{m}$. To do so, we needed to extrapolate the number counts on the faint end. Below $100 \mu \mathrm{Jy}$, the number counts exhibit a powerlaw behavior (Fig. 5). We assumed that this behavior (of the form

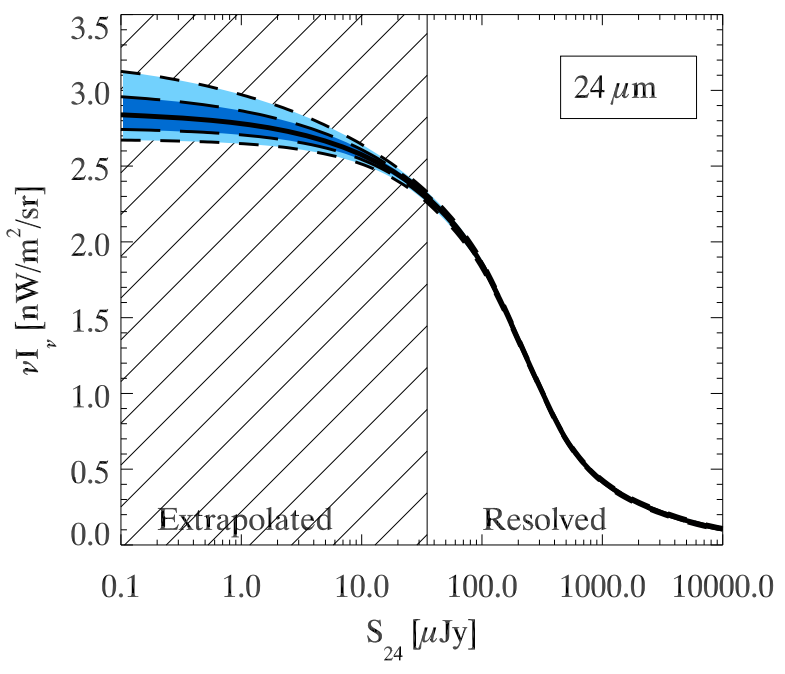

Fig. 9. Cumulative contribution to the surface brightness of the $24 \mu \mathrm{m}$ CIB as a function of $S_{24 \mu \mathrm{m}}$. The colored area represents the $68 \%$ and $95 \%$ confidence level. The shaded area represents the $S_{24}<35 \mu \mathrm{Jy}$ power-law extrapolation zone (see Sect. 6.1). The 4\% calibration uncertainty is not represented. The table corresponding to this figure is available online at http://www.ias.u-psud.fr/irgalaxies/

Table 7. Summary of CIB results found in this article.

\begin{tabular}{llrrr} 
& & $24 \mu \mathrm{m}$ & $70 \mu \mathrm{m}$ & $160 \mu \mathrm{m}$ \\
\hline$S_{\text {cut,resolved }}$ & $\mathrm{mJy}$ & 0.035 & 3.50 & 40.0 \\
$S_{\text {cut,stacking }}$ & & - & 0.38 & 3.1 \\
\hline$v B_{v, \text { resolved }}$ & $\mathrm{nW} \mathrm{m}^{-2} \mathrm{sr}^{-1}$ & $2.29_{-0.09}^{+0.09}$ & $3.1_{-0.2}^{+0.2}$ & $1.0_{-0.1}^{+0.1}$ \\
$v B_{v, \text { resolved+stacking }}$ & & - & $5.4_{-0.4}^{+0.4}$ & $8.9_{-1.1}^{+1.1}$ \\
$v B_{v, \text { tot }}$ & & $2.86_{-0.16}^{+0.19}$ & $6.6_{-0.6}^{+0.7}$ & $14.6_{-2.9}^{+7.1}$ \\
\hline
\end{tabular}

$\left.\mathrm{d} N / \mathrm{d} S=C_{\text {faint }} S^{r}\right)$ still holds below $35 \mu \mathrm{Jy} . r$ and $C_{\text {faint }}$ are determined using the four faintest bins. We found $r=1.45 \pm 0.10$ (compatible with $1.5 \pm 0.1$ of Papovich et al. 2004). Our new estimate of the CIB at $24 \mu \mathrm{m}$ due to infrared galaxies is thus $2.86_{-0.16}^{+0.19} \mathrm{nW} \mathrm{m}^{-2} \mathrm{sr}^{-1}$. The results are plotted in Fig. 9. We conclude that resolved sources down to $S_{24}=35 \mu \mathrm{Jy}$ account for $80 \%$ of the CIB at this wavelength.

\section{2. $70 \mu \mathrm{m}$ and $160 \mu \mathrm{m}$ CIB: lower limit and estimate}

At $70 \mu \mathrm{m}$ and $160 \mu \mathrm{m}$, the integration of the number counts was done in the same way as at $24 \mu \mathrm{m}$, except for the stacking counts, which are correlated. To compute the uncertainties on the integral, we added (on 10000 realizations) a Gaussian error simultaneously to the three quantities and completely recomputed the associated stacking counts: 1 - the mean density flux given by the stacking; 2- the $24 \mu \mathrm{m}$ number counts; 3 - the mean $24 \mu \mathrm{m}$ flux density. At $70 \mu \mathrm{m}$, and $160 \mu \mathrm{m}$, the calibration uncertainty is 7\% (Gordon et al. 2007) and 12\% (Stansberry et al. 2007), respectively. We estimated the CIB surface brightness contribution of resolved sources $\left(S_{70}>3.5 \mathrm{mJy}\right.$ and $\left.S_{160}>40 \mathrm{mJy}\right)$ of $3.1_{-0.2}^{+0.2} \mathrm{nW} \mathrm{m}^{-2} \mathrm{sr}^{-1}$ and $1.0_{-0.1}^{+0.1} \mathrm{nW} \mathrm{m}^{-2} \mathrm{sr}^{-1}$. The contribution of $S_{70}>0.38 \mathrm{mJy}$ and $S_{160}>3.1 \mathrm{mJy}$ is $5.4_{-0.4}^{+0.4} \mathrm{nW} \mathrm{m}^{-2} \mathrm{sr}^{-1}$ and $8.9_{-1.1}^{+1.1} \mathrm{nW} \mathrm{m}^{-2} \mathrm{sr}^{-1}$, respectively.

Below $2 \mathrm{mJy}$ at $70 \mu \mathrm{m}$, and $10 \mathrm{mJy}$ at $160 \mu \mathrm{m}$, the stacking counts are compatible with a power-law. Like at $24 \mu \mathrm{m}$, we assumed that this behavior can be extrapolated and determined the law with the five faintest bins at $70 \mu \mathrm{m}$, and the four faintest at $160 \mu \mathrm{m}$. We found a slope $r=1.50 \pm 0.14$ at $70 \mu \mathrm{m}$, and 


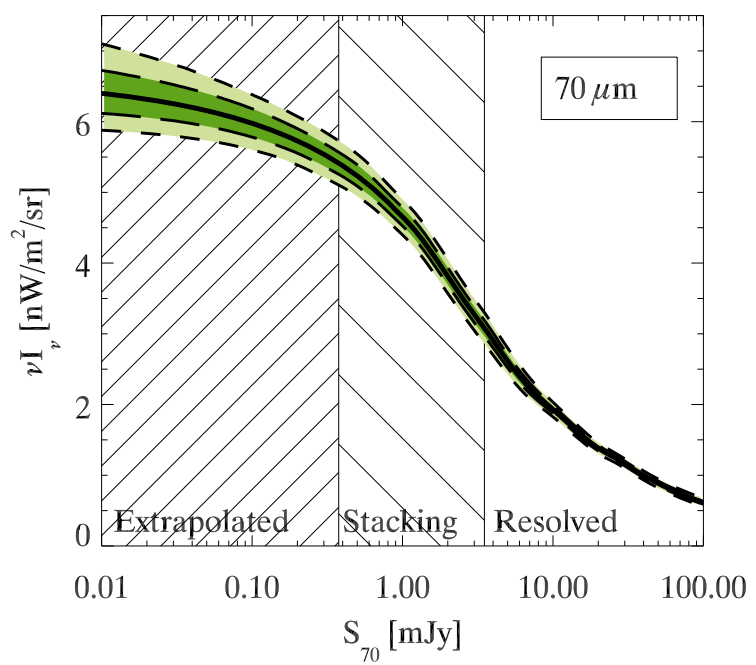

Fig. 10. Cumulative contribution to the surface brightness of the $70 \mu \mathrm{m}$ CIB as a function of $S_{70 \mu \mathrm{m}}$. The colored area represents the $68 \%$ and $95 \%$ confidence level. The shaded areas represent the $0.38<S_{70}<$ 3.3 mJy stacking counts zone and the $S_{70}<0.38$ mJy power-law extrapolation zone (see Sect. 6.2). The 7\% calibration uncertainty is not represented. The table corresponding to this figure is available online at http://www.ias.u-psud.fr/irgalaxies/

$1.61 \pm 0.21$ at $160 \mu \mathrm{m}$. The slope of the number counts at $70 \mu \mathrm{m}$ is compatible with the Frayer et al. (2006) value (1.63 \pm 0.34$)$. The slope at $160 \mu \mathrm{m}$ is measured for the first time. Our new estimate of the CIB at $70 \mu \mathrm{m}$ and $160 \mu \mathrm{m}$ due to infrared galaxies is thus $6.6_{-0.6}^{+0.7} \mathrm{nW} \mathrm{m}^{-2} \mathrm{sr}^{-1}$, and $14.6_{-2.9}^{+7.1} \mathrm{nW} \mathrm{m}^{-2} \mathrm{sr}^{-1}$, respectively. We conclude that resolved and stacking-studied populations account for $82 \%$ and $62 \%$ of the CIB at $70 \mu \mathrm{m}$ and $160 \mu \mathrm{m}$, respectively. These results are summarized in Table 7, and Figs. 10 and 11.

\section{Discussion}

\subsection{New lower limits of the CIB}

The estimations of CIB based on number counts ignore a potential diffuse infrared emission like dust in galaxy clusters (Montier \& Giard 2005). The extrapolation of the faint source counts supposes no low luminosity population, like population III stars or faint unseen galaxies. Accordingly, this type of measurement can provide in principle only a lower limit.

At $24 \mu \mathrm{m}$, Papovich et al. (2004) found $2.7_{1.1}^{-0.7} \mathrm{nW} \mathrm{m}^{-2} \mathrm{sr}^{-1}$ using the counts and the extrapolation of the faint source counts. We agree with this work and significantly reduced the uncertainties on this estimation. Dole et al. (2006) found a contribution of $1.93 \pm 0.23 \mathrm{nW} \mathrm{m}^{-2} \mathrm{sr}^{-1}$ for $S_{24}>60 \mu \mathrm{Jy}$ sources (after dividing their results by 1.12 to correct an aperture error in their photometry at $24 \mu \mathrm{m})$. Our analysis gives $2.10 \pm 0.08 \mathrm{nW} \mathrm{m}^{-2} \mathrm{sr}^{-1}$ for a cut at $60 \mu \mathrm{Jy}$, which agrees very well. Rodighiero et al. (2006) gave a total value of $2.6 \mathrm{nW} \mathrm{m}^{-2} \mathrm{sr}^{-1}$, without any error bar. Chary et al. (2004) found $2.0 \pm 0.2 \mathrm{nW} \mathrm{m}^{-2} \mathrm{sr}^{-1}$, by integrating sources between 20 and $1000 \mu \mathrm{Jy}$ (we find $2.02 \pm 0.10$ for the same interval).

At $70 \mu \mathrm{m}$, using the number counts in the ultra deep GOODS-N and a P(D) analysis, Frayer et al. (2006) found a $S_{70}>0.3 \mathrm{mJy}$ source contribution to the $70 \mu \mathrm{m}$ CIB of $5.5 \pm 1.1 \mathrm{nW} \mathrm{m}^{-2} \mathrm{sr}^{-1}$. Using the stacking counts, we found $5.5 \pm 0.4 \mathrm{nW} \mathrm{m}^{-2} \mathrm{sr}^{-1}$ for the same cut, in excellent agreement and with improved uncertainties. In Dole et al. (2006), the contribution at $70 \mu \mathrm{m}$ of the $S_{24}<60 \mu \mathrm{Jy}$ sources was

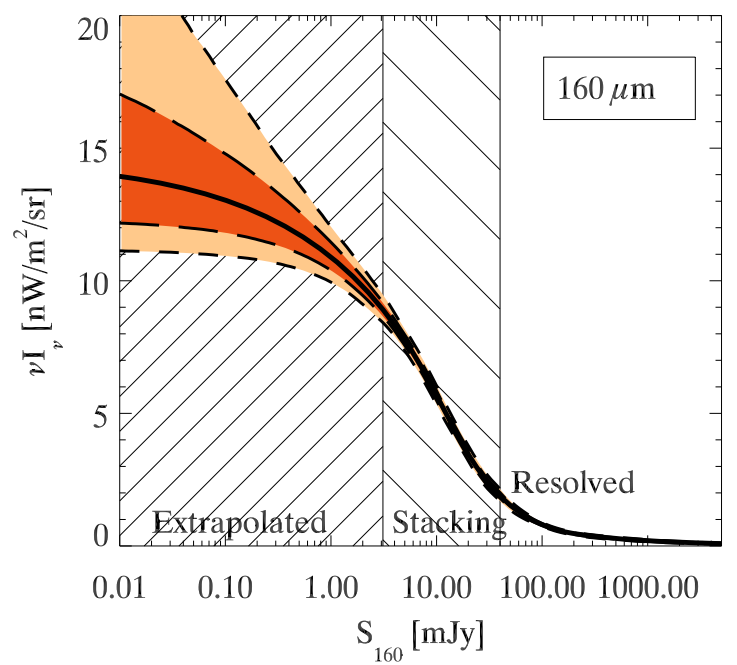

Fig. 11. Cumulative contribution to the surface brightness of the $160 \mu \mathrm{m}$ CIB as a function of $S_{160 \mu \mathrm{m}}$. The colored area represents the $68 \%$ and $95 \%$ confidence level. The shaded areas represent the $3.1<S_{160}<$ 45 mJy stacking counts zone and the $S_{160}<3.1$ mJy power-law extrapolation zone (see Sect. 6.2). The $12 \%$ calibration uncertainty is not represented. The table corresponding to this figure is available online at http://www.ias.u-psud.fr/irgalaxies/

computed with an extrapolation of the $24 \mu \mathrm{m}$ number counts and the $70 / 24$ color. They found $7.1 \pm 1.0 \mathrm{nW} \mathrm{m}^{-2} \mathrm{sr}^{-1}$, but the uncertainty on the extrapolation took only into account the uncertainty on the 70/24 color and not the uncertainty on the extrapolated $24 \mu \mathrm{m}$ contribution, and was thus slightly underestimated. This is in agrees with our estimation.

At $160 \mu \mathrm{m}$ they found with the same method, $17.4 \pm$ $2.1 \mathrm{nW} \mathrm{m}^{-2} \mathrm{sr}^{-1}$ (a corrective factor of 1.3 was applied due to an error on the map pixel size). This estimation is a little bit higher than our estimation, and can be explained by a small contribution (of the order of 15\%) of the source clustering (Bavouzet 2008).

Our results can also be compared with direct measurements made by absolute photometers. These methods are biased by the foreground modeling, but do not ignore the extended emission. Fixsen et al. (1998) found a CIB brightness of $13.7 \pm 3.0$ at $160 \mu \mathrm{m}$, in excellent agreement with our estimation $\left(14.6_{-2.9}^{+7.1} \mathrm{nW} \mathrm{m}^{-2}\right)$. From the discussion in Dole et al. (2006) (Sect. 4.1), the Lagache et al. (2000) DIRBE WHAM (FIRAS calibration) estimation at $140 \mu \mathrm{m}$ and $240 \mu \mathrm{m}$ of $12 \mathrm{nW} \mathrm{m}^{-2} \mathrm{sr}^{-1}$ and $12.2 \mathrm{nW} \mathrm{m}^{-2} \mathrm{sr}^{-1}$ can be also compared with our value at $160 \mu \mathrm{m}$. A more recent work of Odegard et al. (2007) found $25.0 \pm 6.9$ and $13.6 \pm 2.5 \mathrm{nW} \mathrm{m}^{-2} \mathrm{sr}^{-1}$ at $140 \mu \mathrm{m}$ and $240 \mu \mathrm{m}$ respectively (resp. $15 \pm 5.9 \mathrm{nW} \mathrm{m}^{-2} \mathrm{sr}^{-1}$ and $12.7 \pm 1.6 \mathrm{nW} \mathrm{m}^{-2} \mathrm{sr}^{-1}$ with the FIRAS scale). Using ISOPHOT data, Juvela et al. (2009) give an estimation of the CIB surface brightness between $150 \mu \mathrm{m}$ and $180 \mu \mathrm{m}$ of $20.25 \pm 6.0 \pm 5.6 \mathrm{nW} \mathrm{m}^{-2} \mathrm{sr}^{-1}$.

The total brightness due to infrared galaxies at $160 \mu \mathrm{m}$ corresponds to the total CIB level at this wavelength. We thus have probably resolved the CIB at this wavelength. Nevertheless, the uncertainties are relatively large, and other minor CIB contributors cannot be excluded.

In addition, upper limits can be deduced indirectly from blazar high energy spectrum. Stecker \& de Jager (1997) gave an upper limit of $4 \mathrm{nW} \mathrm{m}^{-2} \mathrm{sr}^{-1}$ at $20 \mu \mathrm{m}$ using Mkn 421. Renault et al. (2001) found an upper limit of $4.7 \mathrm{nW} \mathrm{m}^{-2} \mathrm{sr}^{-1}$ between 5 and $15 \mu \mathrm{m}$ with Mkn 501. This is consistent with our lower limit at $24 \mu \mathrm{m}$. 


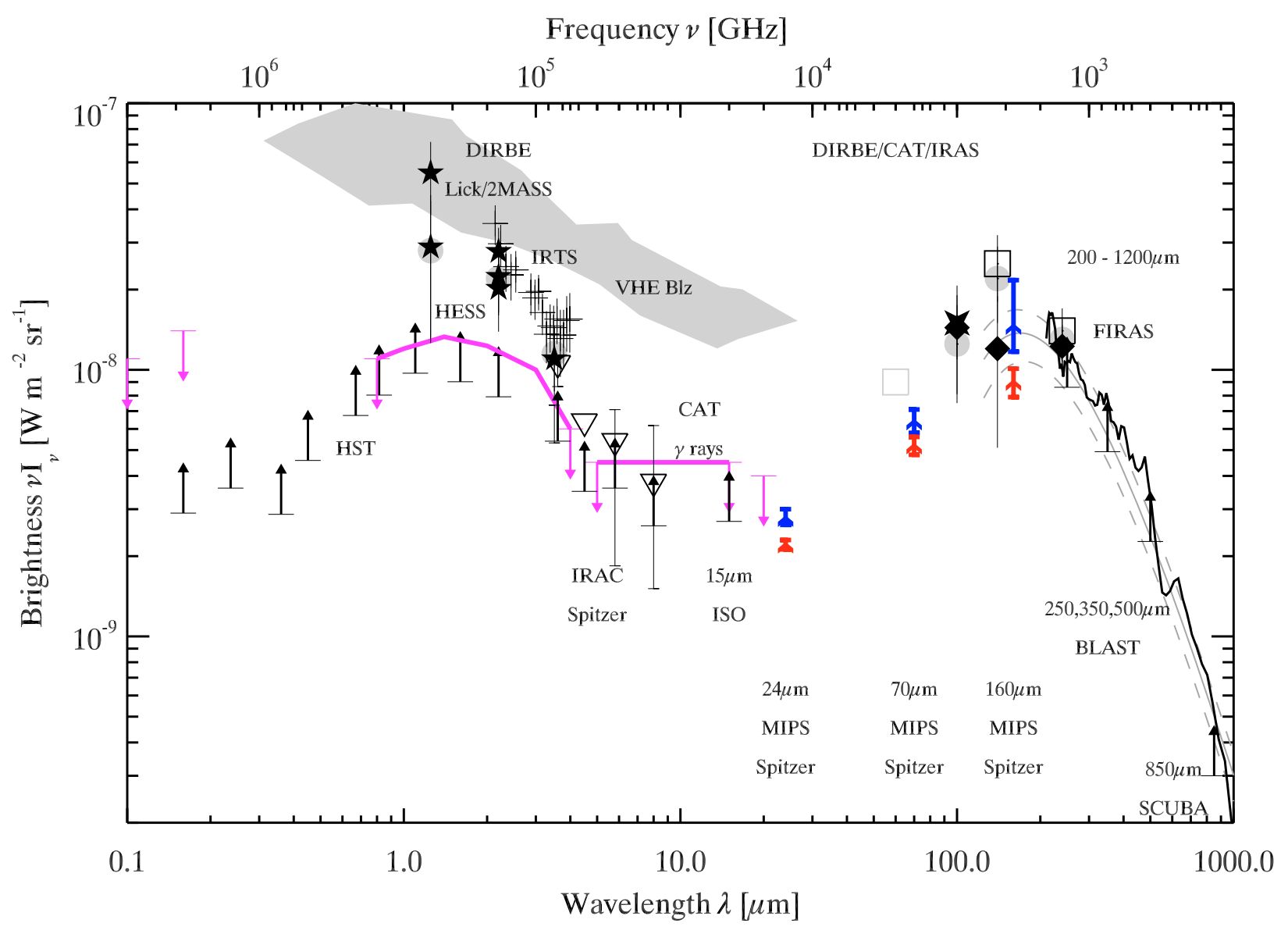

Fig. 12. Current measurement of the extragalactic background light spectral energy distribution from $100 \mathrm{~nm}$ to $1 \mathrm{~mm}$, with the cosmic optical background (COB, $\lambda<8 \mu \mathrm{m}$ ) and cosmic infrared background (CIB, $\lambda>8 \mu \mathrm{m}$ ). Our new points at $24 \mu \mathrm{m}, 70 \mu \mathrm{m}$ and $160 \mu \mathrm{m}$ are plotted (triangle). Lower (red) triangles correspond to the CIB resolved with the number counts and stacking counts. Upper (blue) triangles correspond to the total extrapolated CIB due to infrared galaxies. BLAST lower limits at $250 \mu \mathrm{m}, 350 \mu \mathrm{m}$ and $500 \mu \mathrm{m}$ (Devlin et al. 2009; Marsden et al. 2009) are represented in black arrows. The FIRAS measurements of Fixsen et al. (1998) between $125 \mu \mathrm{m}$ and $2000 \mu \mathrm{m}$ are plotted with a grey solid line, and the $1-\sigma$ confidence region with a grey dashed line. Other points come from different authors (see Dole et al. (2006) for complete details). Old MIPS points are not plotted for clarity.

An update of the synthetic EBL SED of Dole et al. (2006) with the new BLAST (balloon-borne large-aperture submillimeter telescope) lower limits from Devlin et al. (2009) and our values is plotted in Fig. 12. The BLAST lower limits are obtained by stacking of the Spitzer $24 \mu \mathrm{m}$ sources at $250 \mu \mathrm{m}, 350 \mu \mathrm{m}$ and $500 \mu \mathrm{m}$ (Devlin et al. 2009; Marsden et al. 2009).

\section{2. $160 \mu m$ number counts}

At most, we observed a $30 \%$ overestimation of the Lagache et al. (2004) model compared to the $160 \mu$ m number counts (Sect. 4.4 and 5.3 and Fig. 7), despite good fits at other wavelengths. This model uses mean SEDs of galaxies sorted into two populations (starburst and cold), whose luminosity functions evolve separately with the redshift. A possible explanation of the model excess is a slightly too high density of local cold galaxies. By decreasing the density of this local population a little, the model might be able to better fit the $160 \mu$ m number counts without significantly affecting other wavelengths, especially at $70 \mu \mathrm{m}$ (more sensitive to warm dust rather than cold dust), and in the submillimetre range (more sensitive to redshifted cold dust at faint flux densities for wavelengths larger than $500 \mu \mathrm{m}$ ).

The Le Borgne et al. (2009) model slightly overpredicts faint $160 \mu \mathrm{m}$ sources, probably because of the presence of too many galaxies at high redshift; this trend is also seen at $70 \mu \mathrm{m}$. With our number counts as new constraints, their inversion should give more accurate parameters.

Herschel was successfully launched on May 14th, 2009 (together with Planck). It will observe infrared galaxies between $70 \mu \mathrm{m}$ and $500 \mu \mathrm{m}$ with an improved sensitivity. It will be possible to directly observe the cold dust spectrum of high-z ULIRG (ultra luminous infrared galaxy) and medium-z LIRG (luminous infrared galaxy). PACS (Photodetectors Array Camera and Spectrometer) will make photometric surveys in three bands centred on $70 \mu \mathrm{m}, 100 \mu \mathrm{m}$ and $160 \mu \mathrm{m}$. Herschel will allow us to resolve a significant fraction of the background at these wavelengths (Lagache et al. 2003; Le Borgne et al. 2009). SPIRE (spectral and photometric imaging receiver) will observe around $250 \mu \mathrm{m}, 350 \mu \mathrm{m}$ and $500 \mu \mathrm{m}$, and will be quickly confusion limited. In both cases, the stacking analysis will allow us to probe fainter flux density levels, as it is complementary to Spitzer and BLAST.

\section{Conclusion}

With a large sample of public Spitzer extragalactic maps, we built new deep, homogeneous, high-statistics number counts in three MIPS bands at $24 \mu \mathrm{m}, 70 \mu \mathrm{m}$ and $160 \mu \mathrm{m}$. 
At $24 \mu \mathrm{m}$, the results agree with previous works. These counts are derived from the widest surface ever used at this wavelength $\left(53.6 \mathrm{deg}^{2}\right)$. Using these counts, we give an accurate estimation of the galaxy contribution to the CIB at this wavelength $\left(2.86_{-0.16}^{+0.19} \mathrm{nW} \mathrm{m}^{-2} \mathrm{sr}^{-1}\right)$.

At $70 \mu \mathrm{m}$, we used the stacking method to determine the counts below the detection limit of individual sources, by reaching $0.38 \mathrm{mJy}$, allowing us to probe the faint flux density slope of differential number counts. With this information, we deduced the total contribution of galaxies to the CIB at this wavelength $\left(6.6_{-0.6}^{+0.7} \mathrm{nW} \mathrm{m}^{-2} \mathrm{sr}^{-1}\right)$.

At $160 \mu \mathrm{m}$, our counts reached $3 \mathrm{mJy}$ with a stacking analysis. We exhibited for the first time the maximum in differential number counts around $20 \mathrm{mJy}$ and the power-law behavior below $10 \mathrm{mJy}$. We deduced the total contribution of galaxies to the CIB at this wavelength $\left(14.6_{-2.9}^{+7.1} \mathrm{nW} \mathrm{m}^{-2} \mathrm{sr}^{-1}\right)$. Herschel will likely probe flux densities down to about $10 \mathrm{mJy}$ at this wavelength (confusion limit, Le Borgne et al. (2009)).

The uncertainties on the number counts used in this work take carefully into account the galaxy clustering, which is measured with the "counts-in-cells" method.

We presented a method to build very deep number counts with the information provided by shorter wavelength data (MIPS $24 \mu \mathrm{m})$ and a stacking analysis. This tool could be used on Herschel SPIRE data with a PACS prior to probe fainter flux densities in the submillimetre range.

We publicly release on the website http://wWw.ias. $\mathrm{u}$-psud.fr/irgal/, the following products: PSF, number counts and CIB contributions. We also release a stacking library software written in IDL.

Acknowledgements. We wish to acknowledge G. Lagache, who has generated simulations used in this work. We acknowledge J.L. Puget, G. Lagache, D. Marcillac, B. Bertincourt, A. Penin and all members of the cosmology group of IAS for their comments and suggestions. We wish to thank the members of ANR D-SIGALE for their valuable comments, in particular D. Le Borgne for providing us an electronic version of his model. We also thank E. Le Floc'h for quickly providing us the table of his counts. This work is based in part on archival data obtained with the Spitzer Space Telescope, which is operated by the Jet Propulsion Laboratory, California Institute of Technology under a contract with NASA. Support for this work was provided by an award issued by JPL/Caltech. This publication makes use of data products from the Two Micron All Sky Survey, which is a joint project of the University of Massachusetts and the Infrared Processing and Analysis Center/California Institute of Technology, funded by the National Aeronautics and Space Administration and the National Science Foundation.

\section{Appendix A: Uncertainties on number counts including clustering}

\section{A.1. Counts-in-cells moments}

We consider a clustered population with a surface density $\rho$. The expected number of objects in a field of the size $\Omega$ is $\bar{N}=\rho \Omega$. In the Poissonian case, the standard deviation around this value is $\sqrt{N}$. For a clustered distribution, the standard deviation $\sigma_{N}$ is given by (Wall \& Jenkins 2003)

$\sigma_{N}=\sqrt{y \cdot \bar{N}^{2}+\bar{N}}$

The expected value of $y$ is given by (Peebles 1980)

$y=\frac{\int_{\text {field }} \int_{\text {field }} w(\theta) \mathrm{d} \Omega_{1} \mathrm{~d} \Omega_{2}}{\Omega^{2}}$,

where $w(\theta)$ is the angular two points auto correlation function of the sources.

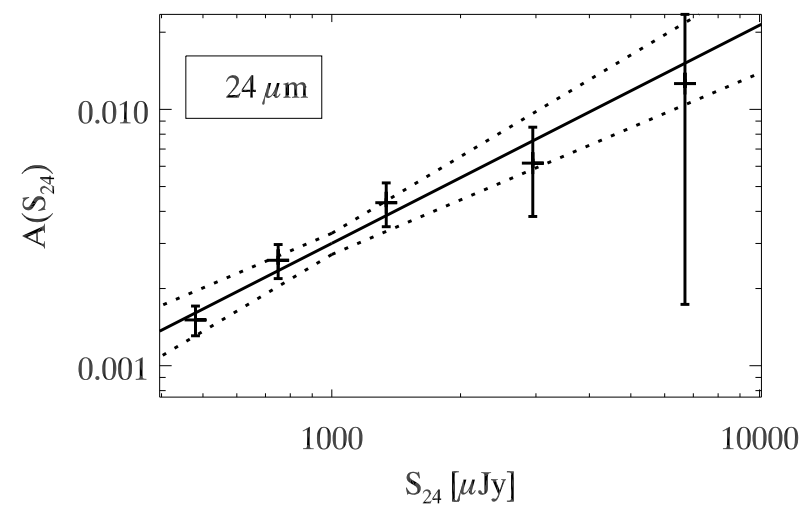

Fig. A.1. Amplitude of the auto correlation as a function of the flux density of the sources at $24 \mu \mathrm{m}$, and best power-law fit.

\section{A.2. Measuring source clustering as a function of flux density}

We assume the classical power law description $w(\theta)=$ $A(S, \lambda) \theta^{1-\gamma}$ with an index $\gamma=1.8$. So, $y$ depends only on $\mathrm{A}$ and on the shape of the field:

$y=A(S, \lambda) \frac{\int_{\text {field }} \int_{\text {field }} \theta^{1-\gamma} \mathrm{d} \Omega_{1} \mathrm{~d} \Omega_{2}}{\Omega^{2}}$.

The uncertainty on $y$ is given by (Szapudi 1998):

$\sigma_{y}=\sqrt{\frac{2}{N_{\text {cell }} \bar{N}^{2}}}$

To measure $A(S, \lambda)$, we cut our fields in $30^{\prime} \times 30^{\prime}$ square boxes, in which we count the number of sources and compute the variance in five, three and three flux density bins at $24 \mu \mathrm{m}, 70 \mu \mathrm{m}$ and $160 \mu \mathrm{m}$. We calculate the associate $A(S, \lambda)$ combining Eqs. (A.1) and (A.3)

$A(S, \lambda)=\frac{\sigma_{N}^{2}-\bar{N}}{\bar{N}^{2}} \times \frac{\Omega^{2}}{\int_{\text {field }} \int_{\text {field }} \theta^{1-\gamma} \mathrm{d} \Omega_{1} \mathrm{~d} \Omega_{2}}$.

The fit of $A\left(S_{24}, 24 \mu \mathrm{m}\right)$ versus $S_{24}$ (see Fig. A.1) gives $\left(\chi^{2}=\right.$ 2.67 for five points and two fitted parameters)

$A(S, 24 \mu \mathrm{m})=(2.86 \pm 0.29) \times 10^{-3}\left(\frac{S}{1 \mathrm{mJy}}\right)^{0.90 \pm 0.15}$.

The measured exponent in A.6 of $0.90 \pm 0.15$ corresponds to $\gamma / 2$, which is the expected value in the case of a fluxlimited survey in an Euclidean universe filled with single luminosity sources. We fix this exponent to fit $A\left(S_{70}, 70 \mu \mathrm{m}\right)$ and $A\left(S_{160}, 160 \mu \mathrm{m}\right)$. We find $A(1 \mathrm{mJy}, 70 \mu \mathrm{m})=(0.25 \pm 0.08) \cdot 10^{-3}$ and $A(1 \mathrm{mJy}, 160 \mu \mathrm{m})=(0.3 \pm 0.03) \cdot 10^{-3}$.

\section{A.3. Compute uncertainties due to clustering}

With this model of $A(S, \lambda)$ and the field shape, we compute $y$ (Eq. (A.3)). Assuming $\bar{N}=N$ (N to be the number of detected sources in a given field and flux density bin), we deduce $\sigma(N)$ from Eq. (A.1), and consequently the error bar on the number counts for a single field.

To compute the final uncertainty on the combined counts, we use the following relation

$\sigma_{\text {comb }, \mathrm{d} N}=\frac{\sqrt{\sum_{i} \Omega_{i}^{2} \sigma_{i, \frac{\mathrm{d} N}{\mathrm{~d} S}}^{2}}}{\sum_{i} \Omega_{i}}$ 


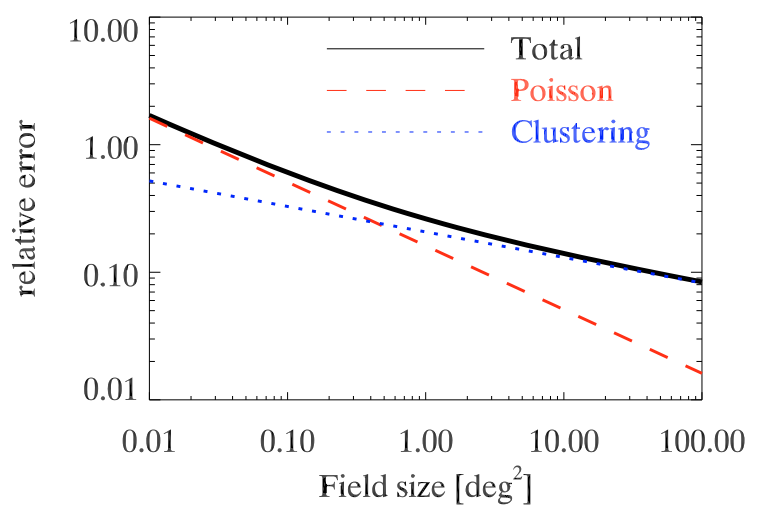

Fig. A.2. Relative error on the number count as a function of the field size. We have chosen $A=0.019$ and $\rho=38.5 \mathrm{deg}^{-2}$ (values for a $80-120 \mathrm{mJy}$ flux density bin at $160 \mu \mathrm{m}$ ). The field is a square.

where $\sigma_{\text {comb, } \frac{\mathrm{d} N}{\mathrm{~d} S}}$ is the uncertainty on the combined number counts, $\Omega_{i}$ the solid angle of the ith field, and $\sigma_{i, \frac{\mathrm{d} N}{\mathrm{~d} S}}$ the uncertainty on the number counts in the ith field (given by $\operatorname{Var}(N)$, Eq. (A.1)).

\section{A.4. Discussion about clustering and number count uncertainties}

For a clustered distribution of sources, the uncertainties on the number counts are driven by two quadratically combined terms (Eq. (A.1)): a Poissonian term $\sqrt{N}$ and a clustering term $\sqrt{y} \cdot N$ (see Fig. A.2). We have $N \propto \Omega$ and $y \propto \Omega^{(\gamma-1) / 2}$ (Blake \& Wall 2002). When the uncertainty is dominated by the Poissonian term (small field), the relative uncertainty is thus proportional to $\sqrt{\Omega^{-1 / 2}}$. When the uncertainty is dominated by the clustering term (large field), the relative error is proportional to $\Omega^{(1-\gamma) / 4}$ $\left(\Omega^{-0.2}\right.$ for $\left.\gamma=1.8\right)$.

Consequently uncertainties decrease very slowly in the the clustering regime. Averaging many small independent fields gives more accurate counts than a big field covering the same surface. For example, in the clustering regime, if a field of $10 \mathrm{deg}^{2}$ has a relative uncertainty of 0.2 , the relative uncertainty is $0.2 / \sqrt{10}=0.063$ for the mean of ten fields of this size, and $0.2 \times 10^{-0.2}=0.126$ for a single field of $100 \mathrm{deg}^{2}$. Consequently, if one studies the counts only, many small fields give better results than one very large field. But, this is not optimal if one studies the spatial properties of the galaxies, which requires large fields.

\section{References}

Arendt, R. G., Odegard, N., Weiland, J. L., et al. 1998, ApJ, 508, 74 Bavouzet, N. 2008, Ph.D. Thesis, Université Paris-Sud 11 http://tel. archives-ouvertes.fr/tel-\$0363975/
Bertin, E., \& Arnouts, S. 1996, A\&AS, 117, 393

Blake, C., \& Wall, J. 2002, MNRAS, 337, 993

Chary, R., Casertano, S., Dickinson, M. E., et al. 2004, ApJS, 154, 80

Devlin, M. J., Ade, P. A. R., Aretxaga, I., et al. 2009, Nature, 458, 737

Diolaiti, E., Bendinelli, O., Bonaccini, D., et al. 2000, A\&AS, 147, 335

Dole, H., Gispert, R., Lagache, G., et al. 2001, A\&A, 372, 364

Dole, H., Lagache, G., \& Puget, J.-L. 2003, ApJ, 585, 617

Dole, H., Le Floc'h, E., Pérez-González, P. G., et al. 2004, ApJS, 154, 87

Dole, H., Lagache, G., Puget, J.-L., et al. 2006, A\&A, 451, 417

Dye, S., Eales, S. A., Ashby, M. L. N., et al. 2006, ApJ, 644, 769

Dye, S., Ade, P. A. R., Bock, J. J., et al. 2009, ApJ, 703, 285

Eddington, A. S. 1913, MNRAS, 73, 359

Eddington, Sir, A. S. 1940, MNRAS, 100, 354

Elbaz, D., Cesarsky, C. J., Chanial, P., et al. 2002, A\&A, 384, 848

Engelbracht, C. W., Blaylock, M., Su, K. Y. L., et al. 2007, PASP, 119, 994

Fernandez-Conde, N., Lagache, G., Puget, J.-L., \& Dole, H. 2008, A\&A, 481, 885

Fixsen, D. J., Dwek, E., Mather, J. C., Bennett, C. L., \& Shafer, R. A. 1998, ApJ, 508,123

Franceschini, A., Rodighiero, G., Vaccari, M., Marchetti, L., \& Mainetti, G. 2010, A\&A, accepted [arXiv:0906.4264]

Frayer, D. T., Huynh, M. T., Chary, R., et al. 2006, ApJ, 647, L9

Frayer, D. T., Sanders, D. B., Surace, J. A., et al. 2009, AJ, 138, 1261

Gispert, R., Lagache, G., \& Puget, J. L. 2000, A\&A, 360, 1

Gordon, K. D., Engelbracht, C. W., Fadda, D., et al. 2007, PASP, 119, 1019

Hauser, M. G., \& Dwek, E. 2001, ARA\&A, 39, 249

Hauser, M. G., Arendt, R. G., Kelsall, T., et al. 1998, ApJ, 508, 25

Juvela, M., Mattila, K., Lemke, D., et al. 2009, A\&A, 500, 763

Kashlinsky, A. 2005, Phys. Rep., 409, 361

Krist, J. 2006, Tiny Tim/Spitzer User's Guide

Lagache, G., Abergel, A., Boulanger, F., Désert, F. X., \& Puget, J.-L. 1999, A\&A, 344, 322

Lagache, G., Haffner, L. M., Reynolds, R. J., \& Tufte, S. L. 2000, A\&A, 354, 247

Lagache, G., Dole, H., \& Puget, J.-L. 2003, MNRAS, 338, 555

Lagache, G., Dole, H., Puget, J.-L., et al. 2004, ApJS, 154, 112

Lagache, G., Puget, J.-L., \& Dole, H. 2005, ARA\&A, 43, 727

Le Borgne, D., Elbaz, D., Ocvirk, P., \& Pichon, C. 2009, A\&A, 504, 727

LeFloc'h, E., Aussel, H., Ilbert, O., et al. 2009, ApJ, 703, 222

Marsden, G., Ade, P. A. R., Bock, J. J., et al. 2009, ApJ, 707, 1729

Montier, L. A., \& Giard, M. 2005, A\&A, 439, 35

Odegard, N., Arendt, R. G., Dwek, E., et al. 2007, ApJ, 667, 11

Papovich, C., Dole, H., Egami, E., et al. 2004, ApJS, 154, 70

Pascale, E., Ade, P. A. R., Bock, J. J., et al. 2009, ApJ, 707, 1740

Pearson, C., \& Khan, S. A. 2009, MNRAS, 399, L11

Peebles, P. J. E. 1980, The large-scale structure of the universe

Puget, J.-L., Abergel, A., Bernard, J.-P., et al. 1996, A\&A, 308, L5

Renault, C., Barrau, A., Lagache, G., \& Puget, J.-L. 2001, A\&A, 371, 771

Rieke, G. H., Young, E. T., Engelbracht, C. W., et al. 2004, ApJS, 154, 25

Rodighiero, G., Lari, C., Pozzi, F., et al. 2006, MNRAS, 371, 1891

Rowan-Robinson, M. 2009, MNRAS, 394, 117

Serjeant, S., Mortier, A. M. J., Ivison, R. J., et al. 2004, ApJS, 154, 118

Shupe, D. L., Rowan-Robinson, M., Lonsdale, C. J., et al. 2008, AJ, 135, 1050

Skrutskie, M. F., Cutri, R. M., Stiening, R., et al. 2006, AJ, 131, 1163

Stansberry, J. A., Gordon, K. D., Bhattacharya, B., et al. 2007, PASP, 119, 1038

Starck, J. L., Aussel, H., Elbaz, D., Fadda, D., \& Cesarsky, C. 1999, A\&AS, 138, 365

Stecker, F. W., \& de Jager, O. C. 1997, ApJ, 476, 712

Stetson, P. B. 1987, PASP, 99, 191

Szapudi, I. 1998, ApJ, 497, 16

Valiante, E., Lutz, D., Sturm, E., Genzel, R., \& Chapin, E. 2009, ApJ, 701, 1814

Wall, J. V., \& Jenkins, C. R. 2003, Practical Statistics for Astronomers

Wang, W.-H., Cowie, L. L., \& Barger, A. J. 2006, ApJ, 647, 74

Werner, M. W., Gallagher, D. B., \& Irace, W. R. 2004, Adv. Space Res., 34, 600 